\title{
A Causal Role for Mouse Superior Colliculus in Visual Perceptual Decision-Making
}

\author{
${ }^{\circledR}$ Lupeng Wang, ${ }^{1}$ Kerry McAlonan, ${ }^{1}$ Sheridan Goldstein, ${ }^{1}$ Charles R. Gerfen, ${ }^{2}$ and ${ }^{\circledR}$ Richard J. Krauzlis ${ }^{1}$ \\ ${ }^{1}$ Laboratory of Sensorimotor Research, National Eye Institute, Bethesda, Maryland 20892, and ${ }^{2}$ Laboratory of Systems Neuroscience, National \\ Institute of Mental Health, Bethesda, Maryland 20892
}

The superior colliculus (SC) is arguably the most important visual structure in the mouse brain and is well known for its involvement in innate responses to visual threats and prey items. In other species, the SC plays a central role in voluntary as well as innate visual functions, including crucial contributions to selective attention and perceptual decision-making. In the mouse, the possible role of the SC in voluntary visual choice behaviors has not been established. Here, we demonstrate that the mouse SC of both sexes plays a causal role in visual perceptual decision-making by transiently inhibiting SC activity during an orientation change detection task. First, unilateral SC inhibition-induced spatially specific deficits in detection. Hit rates were reduced, and reaction times increased for orientation changes in the contralateral but not ipsilateral visual field. Second, the deficits caused by SC inhibition were specific to a temporal epoch coincident with early visual burst responses in the SC. Inhibiting SC during this 100 -ms period caused a contralateral detection deficit, whereas inhibition immediately before or after did not. Third, SC inhibition reduced visual detection sensitivity. Psychometric analysis revealed that inhibiting SC visual activity significantly increased detection thresholds for contralateral orientation changes. In addition, effects on detection thresholds and lapse rates caused by SC inhibition were larger in the presence of a competing visual stimulus, indicating a role for the mouse SC in visual target selection. Together, our results demonstrate that the mouse SC is necessary for the normal performance of voluntary visual choice behaviors.

Key words: decision-making; mouse; perception; superior colliculus; vision; visual attention

\section{Significance Statement}

The mouse superior colliculus (SC) has become a popular model for studying the circuit organization and development of the visual system. Although the SC is a fundamental component of the visual pathways in mice, its role in visual perceptual decision-making is not clear. By investigating how temporally precise SC inhibition influenced behavioral performance during a visually guided orientation change detection task, we identified a $100-\mathrm{ms}$ temporal epoch of SC visual activity that is crucial for the ability of mice to detect behaviorally relevant visual changes. In addition, we found that SC inhibition also caused deficits in visual target selection. Thus, our findings highlight the importance of the SC for visual perceptual choice behavior in the mouse.

\section{Introduction}

The mouse has emerged as a powerful animal model for investigating visual neural circuits (Huberman and Niell, 2011). We might now have more knowledge about the early visual system in mice at molecular, neuronal, and circuit levels than for any

Received Nov. 6, 2019; revised Mar. 15, 2020; accepted Mar. 16, 2020.

Author contributions: L.W. and R.J.K. designed research; L.W., K.M., and S.G. performed research; L.W. and C.R.G. contributed unpublished reagents/analytic tools; L.W. and C.R.G. analyzed data; L.W. and R.J.K. wrote the paper.

The work is supported by the National Eye Institute Intramural Research Program at the National Institutes of Health (ZIA EY000511). We thank N. Nichols and D. Yochelson for technical support.

The authors declare no competing financial interests.

Correspondence should be addressed to Lupeng Wang at lupeng.wang@gmail.com or Richard J. Krauzlis at Richard.krauzlis@nih.gov.

https://doi.org/10.1523/JNEUROSCI.2642-19.2020

Copyright $\odot 2020$ the authors other sensory system in any species (Seabrook et al., 2017), but how mice use visual information to guide voluntary actions has been less studied. Among all visual structures in mice, the superior colliculus (SC) is one of the most important. The mouse SC gets direct inputs from $85 \%$ to $90 \%$ of retinal ganglion cells (RGCs; Ellis et al., 2016) and is also a major output target of the visual cortex (Dräger, 1974). Thus, understanding the functional role of the SC is crucial for understanding vision in mice.

Early visual processing in the SC of mice is important for innate visually guided behaviors, such as avoiding predators (Shang et al., 2015; Wei et al., 2015) and approaching prey (Hoy et al., 2016; Shang et al., 2019). Neurons in the medial SC of the mouse detect looming threats overhead and trigger avoidance behaviors, including escape responses mediated through projections to the dorsal periaqueductal gray (Evans et al., 2018) and 
freezing responses mediated through the basolateral amygdala (Shang et al., 2015; Wei et al., 2015). Lateral SC neurons in mice detect prey items in the lower visual field, and illicit hunting and approach responses through projections to zona incerta (Shang et al., 2019). The SC also sends extensive projections to brainstem and spinal cord motor neurons that generate visually guided orienting responses (Wurtz and Albano, 1980; Dean et al., 1988; Gandhi and Katnani, 2011).

In several other species, the SC also plays a crucial role in voluntary, flexible, and learned visual perceptual functions, such as perceptual decision-making (Boehnke and Munoz, 2008; Herman et al., 2018), visual target selection (Horwitz and Newsome, 1999; Carello and Krauzlis, 2004), and spatial attention (Krauzlis et al., 2013). Lesions or reversible inactivation of the SC can cause behavioral impairments for visual stimuli presented in the affected visual field, such as deficits in saccade generation in primates, seed collecting in hamsters (Schneider, 1969), and nose poking in rats (Dean and Redgrave, 1984). The spatially specific deficits observed in these studies include motor impairments in the ability to orient toward or away from the affected hemifield, in addition to impairments in perceptual processing. This ambiguity can be resolved by using a response that does not involve spatial orienting, for example, by having a monkey press a button or release a joystick (Zénon and Krauzlis, 2012; Lovejoy and Krauzlis, 2017; Herman et al., 2018). Another possibility is to use optogenetic methods to limit the suppression of SC activity to specific epochs of the task, but these methods are not yet reliable in monkeys. In mice, recent work has begun to combine these approaches to identify the role of the SC in visually guided choices (Hu et al., 2019), but clearly distinguishing between changes in visual perceptual versus changes in behavioral strategy remains an ongoing challenge.

Here we investigated the role of mouse SC in perceptual decision-making by training mice to lick a center spout to report the detection of a near-threshold change in visual orientation and used optogenetics to inhibit neuronal activity in the SC during specific temporal windows. We found that unilateral inhibition of SC activity decreased accuracy and increased reaction time for detecting near-threshold orientation changes in the contralateral hemifield but left performance in the ipsilateral hemifield unaffected. These spatially specific deficits were only found consistently when SC activity was inhibited during a 100-ms interval that coincided with transient visual response in the SC. We also measured performance over a range of stimulus amplitudes and constructed psychometric curves so that we could identify changes in visual perceptual processing caused by SC inhibition, distinct from changes in response bias or task engagement. Together, our results demonstrate that visual activity in the mouse SC is crucial for detecting behaviorally relevant events during perceptual decisions.

\section{Materials and Methods}

\section{Animals}

All procedures were conducted on Vgat-ires-Cre mice (JAX stock \#028862, The Jackson Laboratory) and wild-type C57BL/6J mice (stock \#000664, The Jackson Laboratory). Vgat-ires-Cre mice were derived from homozygotes mated with wild-type C57BL/6J mice, producing heterozygote littermates. All of the transgenic animals used in the study were heterozygotes. The mice were housed in a $12 \mathrm{~h}$ reversed day/night cycle, with lights off at 9:00 A.M., and all experimental procedures and behavioral training were performed in the lights-off portion of the cycle (9:00 A.M. to 9:00 P.M.). Male and female mice weighing 18-25 g were surgically implanted at 6-8 weeks of age and then were used in experiments for up to $\sim 9$ months. All the mice were in group housing (2-4 cage mates) before the surgical procedure and subsequently were singly housed after the implant surgery. All experimental procedures and animal husbandry were approved by the National Institutes of Health Institutional Animal Care and Use Committee and complied with Public Health Service policy on the humane care and use of laboratory animals.

\section{Viral vectors}

Double-floxed inverted orientation (DIO) recombinant adeno-associated virus (AAV) vectors (AAV2) were used to express light-gated channel channelrhodopsin-2 [hChR2(H134R)]-enhanced yellow fluorescent protein (eYFP) in Cre-expressing GABAergic neurons in the superior colliculus. The double-floxed inverted hChR2-eYFP cassette was driven with an EF-1a promoter and WPRE (woodchuck hepatitis virus posttranscriptional regulatory element) to enhance expression. The recombinant AAV vector was packaged by the University of North Carolina viral core (titer of $4 \times 10^{12}$ particles $/ \mathrm{ml}$ ).

\section{Stereotaxic surgery}

Each mouse was injected with virus unilaterally and implanted with a head-holder and optic fiber during a single surgical procedure. During the surgery, animals were anesthetized with isoflurane (4\% induction, $0.8-1.5 \%$ maintenance) and were secured by a stereotaxic frame with ear bars (Kopf Instruments). Dexamethasone $(1.6 \mathrm{mg} / \mathrm{kg}$ ) was administered to reduce inflammation. A feedback-controlled heating pad (FHC) was used to maintain the body temperature at $37^{\circ} \mathrm{C}$, and artificial tears were applied to the eyes to prevent them from drying. After the head of the animal was leveled in the stereotaxic frame, a scalp incision was made along the midline, followed by a small craniotomy for virus injection and optic fiber implantation. The coordinates for the unilateral virus injection were $\pm 0.8 \sim 1.1 \mathrm{~mm}$ from midline (medial-lateral axis), -3.65 to approximately $-4.0 \mathrm{~mm}$ from bregma (anterior-posterior axis), and 1.8$2.1 \mathrm{~mm}$ ventral (dorsal-ventral axis), based on a standard mouse brain atlas (Franklin and Paxinos, 2008). Each mouse was injected with 0.1-0.2 $\mathrm{ml}$ of virus at a flow rate of $50 \mathrm{nl} / \mathrm{min}$, using a manual microinjector (Sutter Instrument) with $30-\mu \mathrm{m}$-tip pulled glass pipettes. An optic fiber (200 $\mu \mathrm{m}$ core) together with its ceramic ferrule base (Plexon) were subsequently inserted at the injection coordinates with the fiber tip located at $0.3 \sim 0.5 \mathrm{~mm}$ above the injection center (Fig. $1 \mathrm{~A}$ ). For mice used in recording SC visual responses, a custom 16-wire microwire bundle with Microdrive (Innovative Neurophysiology) was implanted over the cranial window, and lowered into the SC. A custom-designed titanium head post for head fixing was positioned and secured to the skull together with the ferrule using Metabond (Parkell). The skin wound edge was then closed with sutures or tissue adhesive (Vetbond, 3M). After surgery, mice received subcutaneous ketoprofen $(1.85 \mathrm{mg} / \mathrm{kg})$ daily for up to $3 \mathrm{~d}$ to ease discomfort. Fiber placement and viral expression were validated histologically in all mice after completion of data collection.

\section{Food control}

After mice recovered from surgery and returned to $>95 \%$ of their presurgery weight (typically within 7-9d), they were placed on a food control schedule. Mice had free access to water, but their intake of dry food was controlled, and they were allowed to augment their dietary intake by access to a nutritionally complete $8 \%$ soy-based infant formula (Similac). Overall food intake was regulated to maintain at least $85 \%$ of their freefeeding body weight, and the health status of each mouse was monitored daily throughout the study. Mice were initially acclimatized to handling procedures by having their heads gently restrained while receiving the soy-based fluid under manual control via a sipper tube. After the initial exposure to soy-based fluid, we more securely head fixed the animal and continued manual delivery. Once mice were adapted to these procedures, we switched to automatic delivery of fluid under computer control in the behavioral apparatus.

\section{Behavioral apparatus}

The behavioral apparatus consisted of a custom-built booth that displayed visual stimuli to the mouse coupled to their locomotion. The 

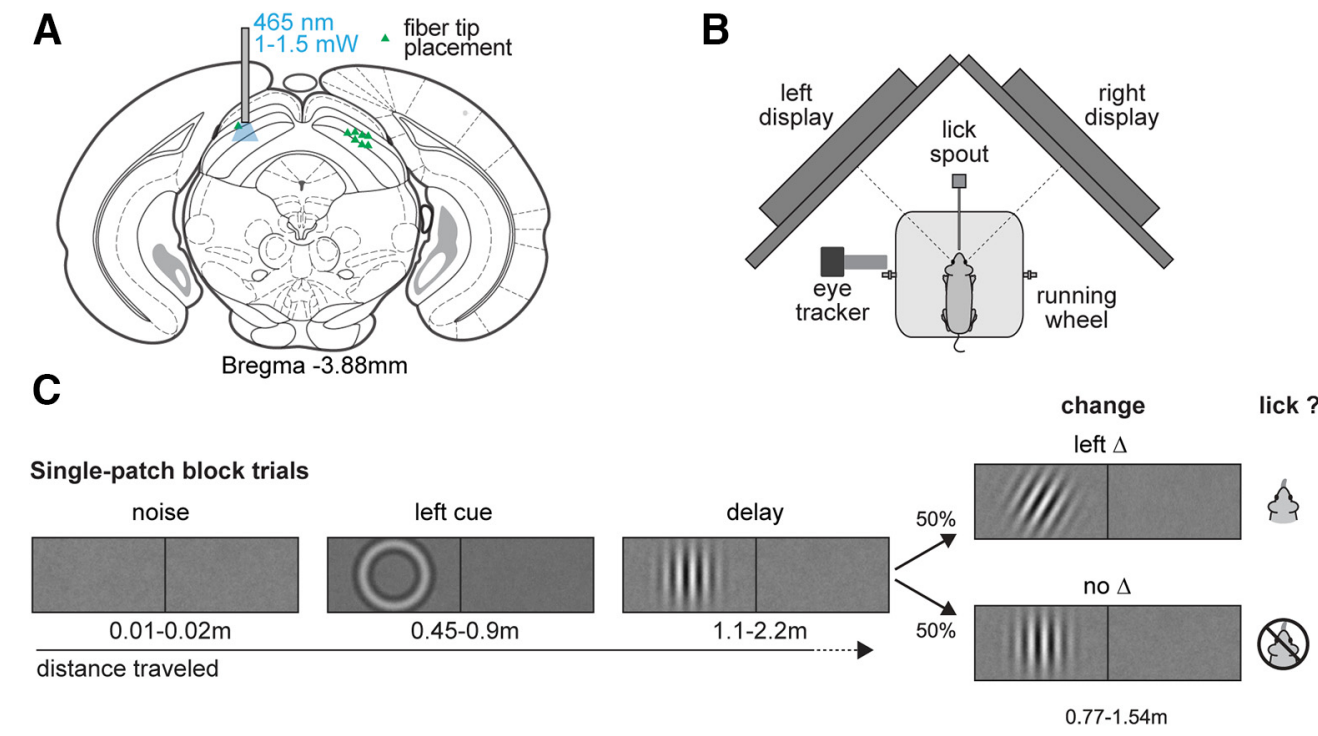

Double-patch block trials

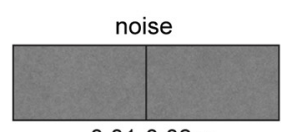

$0.01-0.02 m$

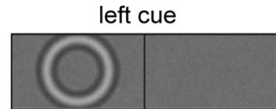

$0.45-0.9 m$
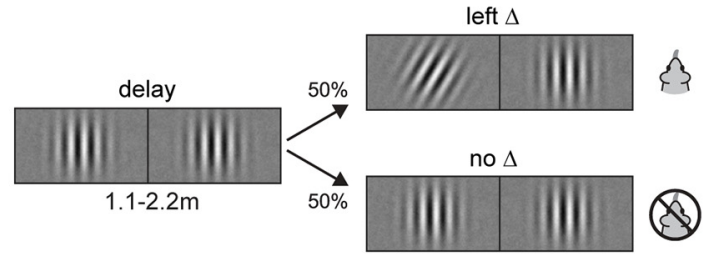

$0.77-1.54 m$

D

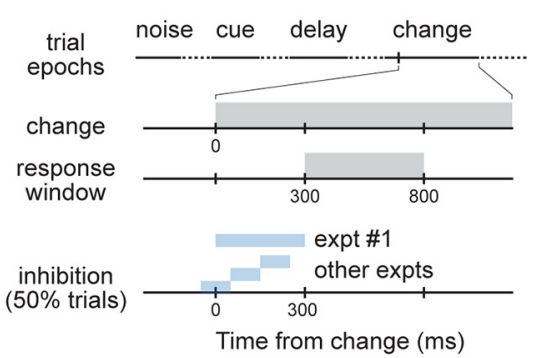

E No-change (catch) trials
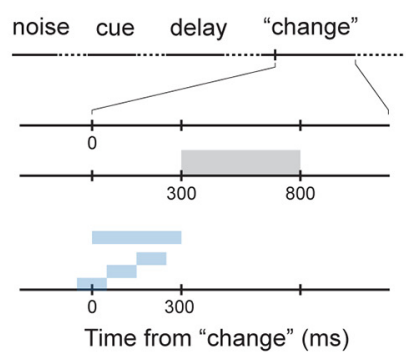

Figure 1. Schematics of orientation change detection task and SC optogenetics stimulation in mice. A, Localization of optogenetic manipulation in the SC, illustrating the stereotactic placement of optical fiber for optical stimulation. $\boldsymbol{B}$, Top-down view of the behavioral apparatus showing the placement of the lickspout, and a head-fixed mouse on a Styrofoam wheel viewing two visual displays. C, Sequence of visual epochs during the detect task for two trial types, illustrating a single-patch (top) and a double-patch (bottom) trial with left-side orientation change. The length of each epoch was defined as the distance the mouse traveled through the trial. $\boldsymbol{D}, \boldsymbol{E}$, Timeline of events and response windows in change $(\boldsymbol{D})$ and no-change $(\boldsymbol{E})$ trials. Trial outcomes were defined based on a 500-ms response window for licks, which started $300 \mathrm{~ms}$ after the orientation change; extraneous licks triggered trials to abort. The optogenetic stimulation window varied across experimental conditions, but all ended before the beginning of response window. Blue shading indicates the timing of SC inhibition applied during the experiments.

mouse was head fixed in the center of the apparatus, positioned atop a polystyrene foam wheel $(20 \mathrm{~cm}$ diameter) that allowed natural walking or running movements along a linear path. An optical encoder (Kübler) was used to measure the rotation of the wheel. The front walls of the booth incorporated a pair of LCD displays (model VG2439, ViewSonic) positioned at $45^{\circ}$ angles from the midline of the animal such that each display was centered on either the right or left eye and subtended $\sim 90^{\circ}$ horizontal by $\sim 55^{\circ}$ vertical of the visual hemifield, with a viewing distance of $27.5 \mathrm{~cm}$. The interior of the booth was lined with sound-absorbent material to reduce acoustic noise. The experiments were controlled by a computer using a modified version of the PLDAPS system (Eastman and Huk, 2012). Our system omitted the Plexon device, but included a Datapixx peripheral (Vpixx Technologies) and the Psychophysics Toolbox extensions (Brainard, 1997; Pelli, 1997) for MATLAB (MathWorks), controlled by MATLAB-based routines run on a Mac Pro (Apple). The Datapixx device provided autonomously timed control over analog and digital inputs and outputs, and synchronized the display of visual stimuli generated using the Psychophysics Toolbox. A reward delivery spout was positioned near the snout of the mouse; lick contacts with the spout were detected by a piezo sensor (Mide Technology) and custom electronics. Each reward was a small volume $(5-10 \mu \mathrm{l})$ of an $8 \%$ solution of soy-based infant formula (Similac) delivered by a peristaltic pump (Harvard Apparatus) under computer and Datapixx control. Airpuff aversive stimuli were delivered through a second spout located slightly above the reward spout, and controlled through solenoids (Parker Hannifin). The temperature inside the apparatus maintained between 70 and $80^{\circ} \mathrm{F}$.

\section{Visual detection tasks}

The detection task was modified from our previously published study (Wang et al., 2018). Animals were run in experiments on consecutive days, and each session produced 300-900 trials. Experiments were organized in blocks of randomly shuffled, interleaved trials, and each trial consisted of a sequence of epochs that the mouse passed through by walking or running on the wheel. Each epoch was defined by the particular stimuli presented on the visual displays, and the duration of each epoch was determined by the time that it took for the mouse to travel a 
randomized distance on the wheel, typically several seconds based on the running speed of the mice (median, $85.6 \mathrm{~cm} / \mathrm{s}$ ).

In all of the experiments, each trial followed a standard sequence of four epochs. The average luminance across each visual display in all epochs was $4-8 \mathrm{~cd} / \mathrm{m}^{2}$. In the first epoch ("noise"), the uniform gray of the intertrial interval was changed to pink visual noise with a root mean square contrast of $3.3 \%$; this epoch was presented for a distance of $0.9-$ $1.8 \mathrm{~cm}$ (i.e., $0.007-0.03 \mathrm{~s}$ ). In the second epoch ("cue"), a contrast annulus was added to the pink noise, center in either left or right visual display. The annulus consisted of a sinusoidal concentric function, with radius and width matched to the Gabor patch subsequently shown. This second epoch lasted for $46-92 \mathrm{~cm}(0.36-1.55 \mathrm{~s})$. In the third epoch ("delay"), it depended on the trial type. If it was a single-patch trial, a vertically oriented Gabor patch was added to the pink noise, centered in either the left or right visual display. The Gabor patch consisted of a sinusoidal grating (95\% Michelson contrast) with a spatial frequency of 0.1 cycles per degree, a value chosen based on the visual spatial acuity of mice (Sinex et al., 1979), modulated by a Gaussian envelope with fullwidth at half-maximum of $18^{\circ}$ (standard deviation $(\mathrm{SD})=7.5^{\circ}$ ). The phase of the grating was not fixed, but throughout the trial was incremented in proportion to the wheel rotation with every monitor refresh, so that the sinusoidal pattern was displaced on the screen by approximately the same distance that the mouse traveled on the wheel; the Gabor patch on the left (right) drifted leftward (rightward), consistent with optic flow during locomotion. If it was a double-patch trial, two Gabor patches with the same properties as in single-patch trials appeared on the opposite sides of the visual displays. This third epoch lasted for $107-214 \mathrm{~cm}(0.84-3.6 \mathrm{~s})$. The visual stimuli in the fourth epoch depended on whether the trial was a "change" or "no-change" condition; the two types were equally likely and randomly interleaved within a block, and both lasted 77-154 cm (0.61-2.6 s). On single-patch change trials, the Gabor patch changed its orientation at the onset of the fourth epoch. On double-patch change trials, the Gabor patch on the same side as the cue ring changed its orientation at the onset of the fourth epoch. The direction of the orientation change observed mirror symmetry, as follows: if the left (right) Gabor changed, it rotated clockwise (counterclockwise). On no-change trials, neither Gabor patch changed its orientation, so that the fourth epoch unfolded as a seamless extension of the previous two-patch epoch.

The task of the mouse was to lick the spout when he or she detected a change in the orientation of the Gabor patch and to otherwise withhold from licking. Mice were required to lick within a 500-ms response window starting $300 \mathrm{~ms}$ after the orientation change to score a "hit" and receive a fluid reward. The initial $300 \mathrm{~ms}$ no-lick period was applied to most mice, except for a few mice that had faster reaction times, for whom the response windows were shifted earlier by $50 \mathrm{~ms}$. If the mouse failed to lick within this window after an orientation change, the trial was scored as a "miss" and no reward was given but no other penalty was applied. On no-change trials, if the mouse licked within the same response window aligned on the transition to epoch 4, the trial was scored as a "false alarm"; if they correctly withheld from licking, the trial was scored as a "correct reject." At the end of correct reject trials, the trial was extended to include an additional "safety-net epoch" in which the initially appearing Gabor underwent a suprathreshold $\left(30^{\circ}\right)$ orientation change, and the mouse could receive a reward by licking within a comparable response window. The point of this safety net epoch was to maintain motivation by rewarding the mouse for correct behavior without violating the task rule that they should lick only for orientation changes. False alarms and premature licks before the response window led to timeouts and possible air-puff penalties; well trained mice usually committed $<10 \%$ of such errors.

All trials followed the standard four-epoch trial sequence and were organized into alternating blocks in which the orientation change occurred in either the left or right Gabor patch. We counterbalanced the frequency of trials with and without orientation changes, and with and without stimulation, to minimize possible behavioral biases related to frequency matching. Each block of trials with cue on the same side included two sub-blocks, started with 60 single-patch trials followed with 60 double-patch trials. Blocks of left and right cue trials were randomly interleaved within each experimental session.
For experiments with 300-ms SC inhibition, we used a single value of $15^{\circ}$ for the orientation change. For experiments with orange LED stimulation, we used a $12^{\circ}$ orientation change. Each of the four possible combinations of two factors (change/no-change, with/without SC inhibition) comprised $25 \%$ of the total trials in each block.

For experiments with three epochs of SC inhibition, we used a single value of $12^{\circ}$ for the orientation change. Each sub-block of 60 trials with cue on the same side contained $25 \%$ trials with orientation change and no inhibition, $25 \%$ trials without orientation change and no inhibition, $25 \%$ trials with orientation change and SC inhibition drawn equally from three epochs, and $25 \%$ trials without orientation change and with inhibition from the same three epochs. All different trial types within the same sub-block were randomly interleaved.

For the psychometric curve experiments, each sub-block of 60 trials contained $25 \%$ with an orientation change drawn equally from five possible values $\left(4^{\circ}, 7^{\circ}, 11^{\circ}, 15^{\circ}\right.$, and $\left.20^{\circ}\right)$ and with no inhibition, $25 \%$ with the same five orientation changes with SC inhibition during epoch 2, $25 \%$ with no change and no inhibition, and $25 \%$ with no change and inhibition during epoch 2. Because only some of the orientation changes could be reliably detected, a safety net epoch was added to the end of miss trials, but with a long duration to discourage using it as a default response. We recorded 8-10 sessions for each mouse to obtain at least 50 repetitions for each of the five orientation changes. The experimenters were blind to the side of SC inhibition of animals, and the order of presentation for the different trial types in each experiment was also randomized.

\section{Electrophysiological recording}

In a set of mice separate from the optogenetic manipulations, we obtained electrophysiological recordings from isolated neurons in the SC. The purpose of these recordings was to document the time course of firing rate modulation during the task to guide our choices about the temporal epochs for SC inhibition. The activity of SC neurons was recorded in two male C57BL/6J mice implanted with a moveable 16-wire microwire bundle (Innovative Neurophysiology). Electrophysiological signals were acquired through an RZ5D processor (Tucker-Davis Technologies) with spikes filtered between 0.3 and $7 \mathrm{kHz}$ and sampled of $25 \mathrm{kHz}$. Single units were sorted offline using KiloSort (Pachitariu et al., 2016). The SC surface was determined as the first visual responses encountered while advancing the microwire bundle. All single-unit data were collected from depths within $2 \mathrm{~mm}$ from the estimated SC surface. A total of 260 isolated single units recorded across 40 individual sessions were included in the analysis, regardless of their selectivity to task epochs. Firing rates of each neuron were summarized by counting spikes within 10-ms-wide nonoverlapping bins aligned on the time of the orientation change, and then normalizing the value within each bin by the maximum count during the interval starting $300 \mathrm{~ms}$ before the change until $800 \mathrm{~ms}$ after the change.

\section{Monitoring eye movements}

A high-speed, $240 \mathrm{~Hz}$ CCD camera (ISCAN) was used to monitor the eye position of head-fixed mice during the three-epoch SC inhibition experiments. We imaged an area of $1.5 \times 3 \mathrm{~mm}$ with a macro lens (ISCAN) centered on the eye. Four infrared light-emitting diodes (LEDs; $940 \mathrm{~nm}$ ) were used to illuminate the eye. Commercially available acquisition software (ETL-200, ISCAN) was used to determine the center and boundary of the pupil. Eye position was obtained by subtracting the center of corneal reflection from the pupil center to compensate any translational movement of the eye at the imaging plane. The pupil displacement in 2-D image was converted to a rotation angle based on estimated eyeball radius $(1.25 \mathrm{~mm}$ ) from model C57BL/6 mice (Sakatani and Isa, 2004; Stahl, 2004). Eye velocity was then obtained by applying a low-pass differentiating filter to eye position traces $(-3 \mathrm{~dB}$ rolloff at $54 \mathrm{~Hz}$ ). Saccade detection was performed in a custom graphic user interface written in MATLAB script, following a previously described algorithm (Krauzlis and Miles, 1996); thresholded at a minimum velocity of $70 \%$ s, minimum acceleration of $3000 \% \mathrm{~s}^{2}$, and a minimum duration of 15 $\mathrm{ms}$; and all eye traces were manually inspected to remove blink artifacts. Saccade probability was calculated as the fraction of trials within each time bin that were marked as containing a saccade. 


\section{Histology}

Mice were killed with $\mathrm{CO}_{2}(1.0 \mathrm{~L} / \mathrm{min})$, after which they were transcardially perfused with ice-cold saline followed by PBS with $4 \%$ paraformaldehyde (PFA). Their brains were removed and stored in the 4\% PFA solution overnight, and then transferred to a PBS with $20 \%$ sucrose solution for at least $3 \mathrm{~d}$ before sectioning. The $80 \mu \mathrm{m}$ frozen sections were cut coronally using a freezing microtome, and free-floating sections were processed for immunohistochemical labeling of GFP mounted on gelatin-coated glass slide and counterstained with a fluorescent blue counterstain. To visualize the injection site and axonal projections from viral transfected cells, sections were imaged at $10 \times$ using a Zeiss fluorescent microscope using Neurolucida software (MBF Bioscience) to produce a whole-brain reconstruction from tiled images of coronal or sagittal sections.

Density maps of the areal extent and depth of viral expression were obtained using the ImageJ image-processing package (National Institutes of Health) based on the stacks of coronal histology sections through the SC. To estimate the areal extent of expression across the SC, intensity values were averaged across $\mathrm{SC}$ depth and projected onto a horizontal-plane view for all sections, using the top/down reslice and $z$-projection functions in ImageJ. The raw expression density maps were then smoothed by applying Gaussian blur with an SD of $80 \mu \mathrm{m}$. Contour lines at four intensity levels $(20 \%, 40 \%, 60 \%$, and $80 \%)$ were obtained using IsoPhotContour2 plugin in ImageJ from the smoothed density maps. Finally, the resulting horizontal density maps were aligned with the SC retinotopic visual map (Drager and Hubel, 1976) based on anatomic landmarks. To estimate the expression across different SC layers, a similar smoothing and contour-line drawing procedure was applied to the coronal section from each mouse at the anterior/posterior location corresponding to the peak of the horizontal density map.

\section{Experimental design and statistical analysis}

Data came from a total number of 10 mice in the study. Eight mice were Vgat-ires-Cre, and two mice were C57BL/6J. Among the eight Vgat-iresCre mice used in the study, five were male and three were female; the two C57BL/6J mice were male. We did not observe any systematic difference in behavioral performance between sexes in this study. Four mice (Vgat-ires-Cre) were used for studying the effects of 300-ms SC inhibition on detection performance; among them, two were males, and the other two were females. The two C57BL/6J mice were used for electrophysiological recordings. Eight Vgat-ires-Cre mice were used for studying the effects of three 100-ms temporal epochs of SC inhibition on detection performance, and the same eight mice were used for studying the effects of SC inhibition on psychometric curves. Five Vgat-ires-Cre mice were used for studying the effects of orange light stimulation on detection performance, two were female and three were male. Five Vgatires-Cre mice were used for eye tracking while with SC inhibition; two were female and three were male.

For the psychometric data, results were pooled across sessions for each mouse to tabulate the overall lick probability for each amplitude of orientation change (including no change) separately for left and right visual fields and for with and without optogenetic stimulation. The lick probabilities were fitted with a cumulative Gaussian function using Psignifit3 (Wichmann and Hill, 2001). The fitted function had the following four parameters: mean, SD of the Gaussian, bias (lower asymptote), and lapse rate (upper asymptote). Based on the SD, we calculated the just-noticeable difference (JND), defined as the SD multiplied by $\sqrt{ } 2$; the JND indicates the minimum change in signal strength that increases the detection rate by at least $50 \%$ of the available response range, once response bias and lapse rate have been taken into account.

For experiments using a single value of orientation change, we also pooled across sessions to tabulate lick probability, and tabulated hit and false alarm rates based on the definitions of trial outcomes described for the behavioral tasks. Performance was then characterized by measuring sensitivity $\left(d^{\prime}\right)$ and criterion using methods from signal detection theory (Macmillan and Creelman, 2005), as follows: $d^{\prime}=\Phi^{-1}(\mathrm{H})-\Phi^{-1}(F)$, criterion $=-\Phi^{-1}(\mathrm{H})+\Phi^{-1}(F) / 2$, where $\Phi^{-1}$ is the inverse of normal cumulative distribution function, $H$ is the hit rate, and $F$ is the false alarm rate. The $95 \%$ confidence intervals (CIs) of hit and false alarm rates were generated with the binofit function in MATLAB, which uses the Clopper-Pearson method to calculate confidence intervals. The $95 \%$ confidence intervals of $d^{\prime}$ and criterion were generated with bootstrapped resampling.

Statistical analyses were conducted in MATLAB using the statistics and machine learning toolbox and Prism 7 (GraphPad Software), and statistical significance was accepted as $p<0.05$. Paired-sample nonparametric Wilcoxon signed-rank tests were performed to compare the cross-subject effect of SC inhibition on hit rate, false alarm rate, reaction times, $d^{\prime}$, and criterion across the population of mice, unless stated otherwise. $\chi^{2}$ tests were performed to compare the within-subject effect of SC inhibition on hit rate. Nonparametric Wilcoxon rank sum tests were performed to compare the cross-subject effect of 300-ms SC inhibition on hit rate and reaction times, and the within-subject effect of SC inhibition on reaction times. Two-way ANOVAs were used to assess the interaction between SC inhibition during epoch 2 and spatial location for reaction times, using inhibition versus no inhibition and contralateral versus ipsilateral as two independent factors, the interactions were further tested with post hoc multiple comparisons using the Bonferroni method. Paired-sample nonparametric Wilcoxon signed-rank tests were performed to compare the across-subject effect of SC inhibition on fitting parameters of psychometric curves (threshold, JND, lapse rate, guess rate). Nonparametric Wilcoxon signed-rank tests were performed to compare the cross-subject effect of stimulus competition on the magnitude of SC inhibition-caused changes in fitting parameters of psychometric curves (threshold, JND, lapse rate, guess rate). The value of $n$ reported in the figures and results indicates the number of animals. Error bars in figures indicate the $95 \%$ confidence interval of the median or mean, unless otherwise indicated.

\section{Code accessibility}

All of the data were acquired and initially processed using custom scripts written in MATLAB (MathWorks). The MATLAB code and data that support the findings of this study will be made available from the corresponding author on reasonable request.

\section{Results}

\section{SC inhibition caused deficits in change detection for contralateral hemifield}

Head-fixed mice were trained to detect near-threshold changes in the orientation of vertical Gabor patches centered in the left and right visual displays (Fig. $1 B$ ), using a task described previously (Wang et al., 2018; Wang and Krauzlis, 2018). Each trial was self-initiated, and mice progressed through a sequence of epochs based on a randomized distance traveled on the wheel (Fig. 1C). On change trials (Fig. 1D), mice scored a "hit" if they reported their detection by contacting the lickspout within a 500-ms window starting $300 \mathrm{~ms}$ after the change event. On an equal number of interleaved no-change trials (Fig. 1E), mice were assessed a "false alarm" if they contacted the lickspout in the absence of the orientation change.

The causal role of SC in visual detection performance on this task was investigated by unilaterally inhibiting neuronal activity in the SC, achieved by targeted optogenetic activation of GABAergic inhibitory neurons (Fig. 1A) during various temporal intervals before the response window (Fig. 1D). The effectiveness of this optogenetic strategy has been previously demonstrated in the SC (Rossi et al., 2016; Ahmadlou et al., 2018), thalamus (Guo et al., 2017), and visual cortex (Liu et al., 2016); we will refer to this manipulation as "SC inhibition" throughout the study. The areal extent and layers of the SC affected by this manipulation in our mice were estimated by histologic reconstruction of viral expression (Fig. 2). The affected portions of the SC included large fractions of the retinotopic representation of the contralateral visual field, overlapped the portion of the visual field occupied by the 
A
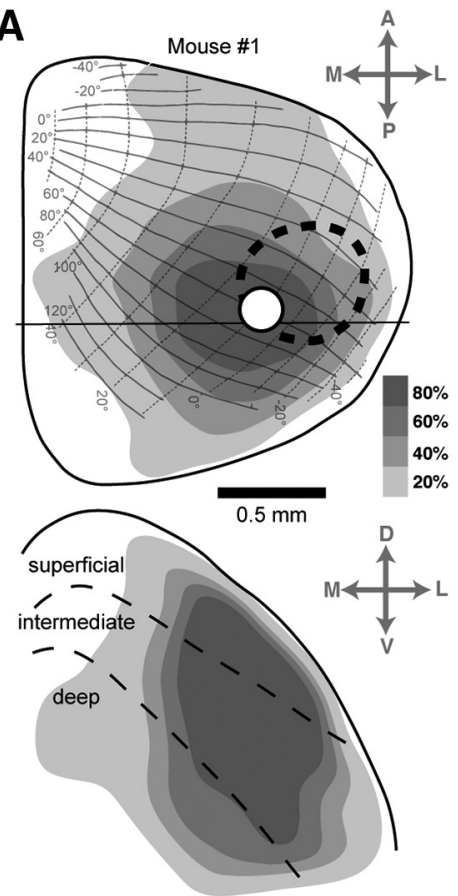

Bregma: $-4.08 \mathrm{~mm}$

E

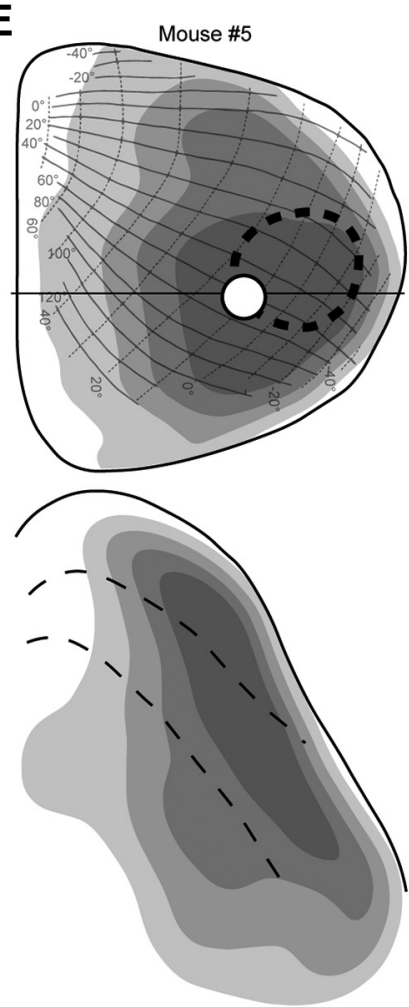

Bregma: $-3.94 \mathrm{~mm}$
B
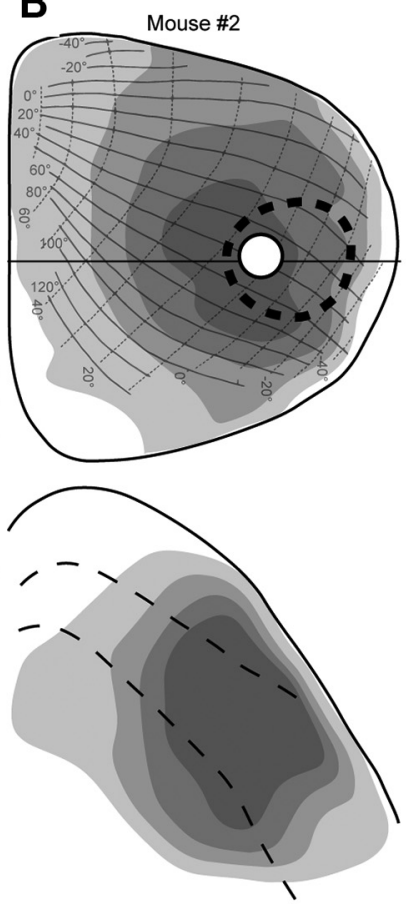

Bregma: -3.86mm
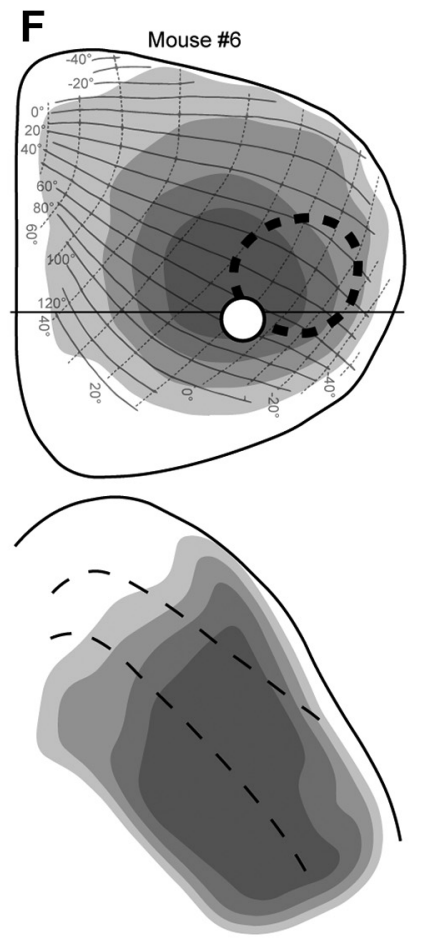

Bregma: $-4.04 \mathrm{~mm}$
C
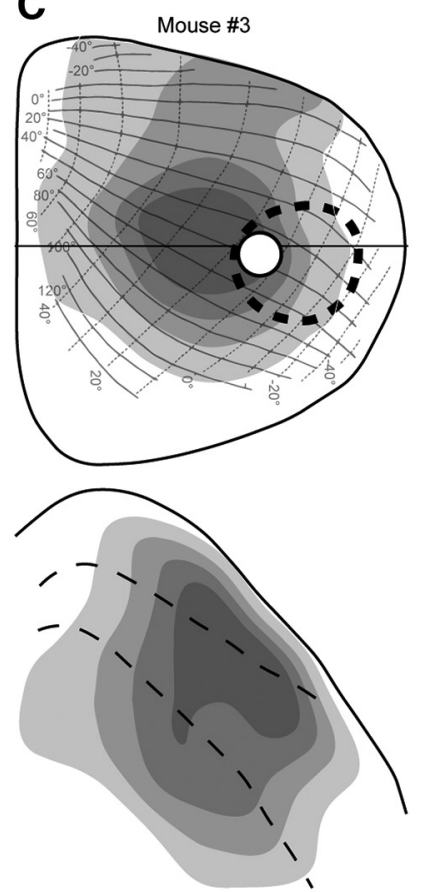

Bregma: $-3.78 \mathrm{~mm}$

G
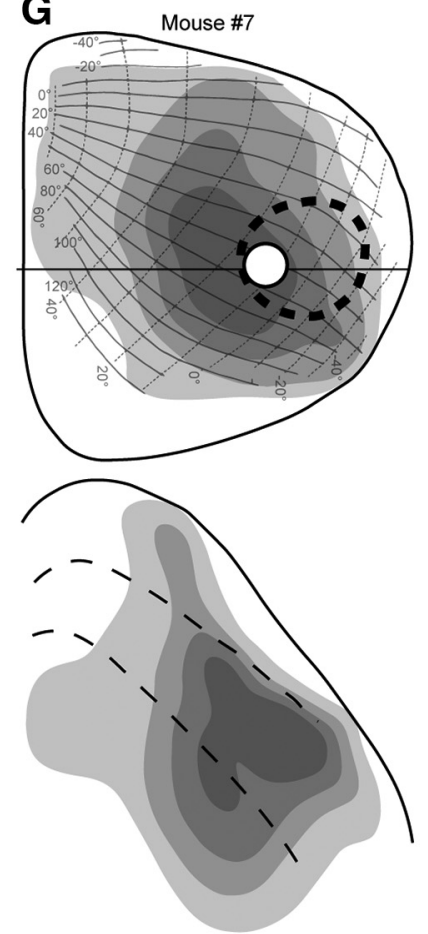

Bregma: $-3.90 \mathrm{~mm}$
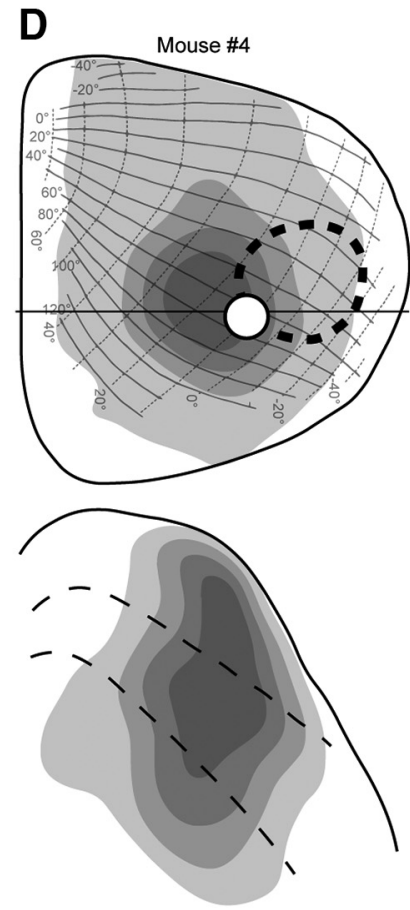

Bregma: $-4.00 \mathrm{~mm}$
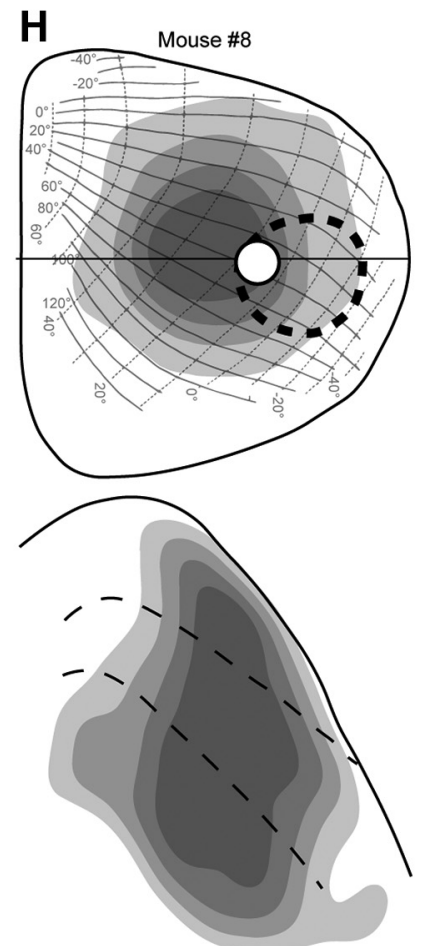

Bregma: $-3.78 \mathrm{~mm}$

Figure 2. Areal extent and depth of $S C$ tissue affected in each mouse. $A$, Density map of viral expression in the SC of mouse 1. Top, Density of viral expression projected onto horizontal plane view of the SC, with overlay of the SC retinotopic map (adapted from Drager and Hubel, 1976). The levels of gray shading indicate the density of viral expression as a percentage of the maximum possible value. Dashed oval indicates the retinotopic area of the SC map occupied by the Gabor patch visual stimulus. White circle indicates the location of the optic fiber. Black horizontal line indicates the coronal section at the epicenter of the injection, illustrated in the bottom panel. Scale bar, $500 \mu \mathrm{m}$. Bottom, Density map of viral expression in the coronal section at the epicenter of injection in mouse 1. Solid line depicts SC upper surface, and dashed lines demarcate superficial, intermediate and deep layers of SC. B-H, Density maps of viral expression in the other seven mice used in the experiment. Conventions are the same as for mouse 1 in $A$. For mouse 7, the left $\mathrm{SC}$ was injected, but the data are presented mirror reversed for ease of comparison with the other cases. 
A
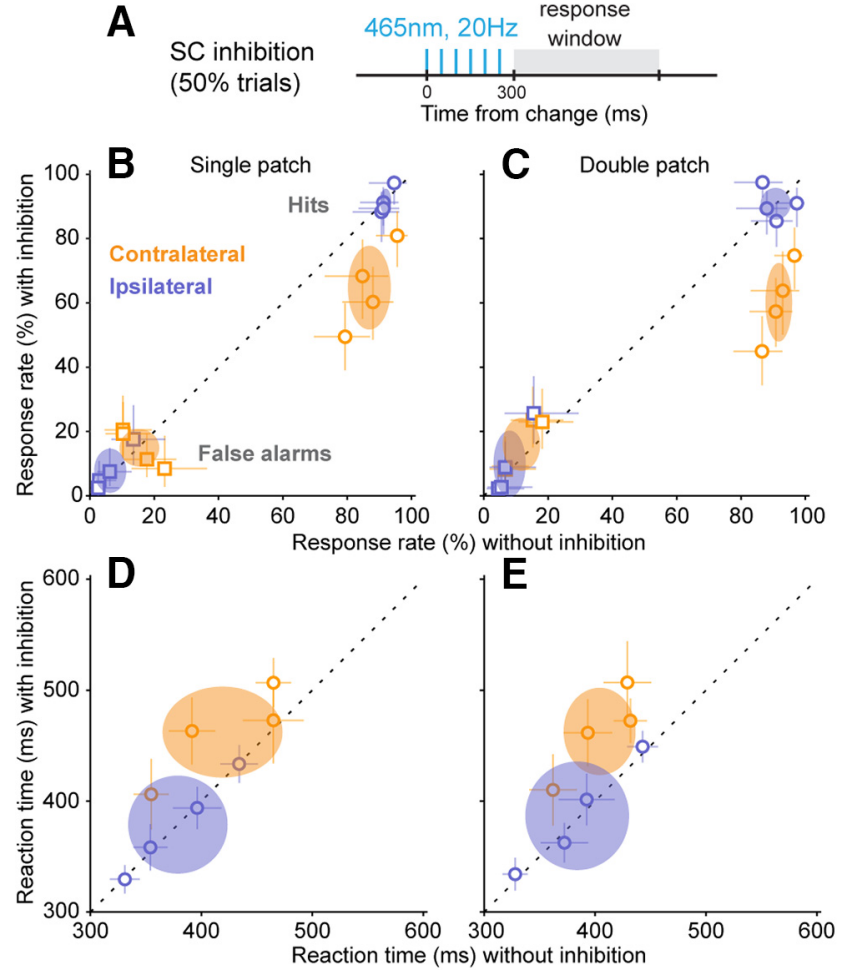

Figure 3. Unilateral $\mathrm{SC}$ inhibition impaired detection of contralateral orientation changes. $\boldsymbol{A}$, Timing of the $300-\mathrm{ms}$ optogenetic stimulation with respect to the orientation change. $\boldsymbol{B}$, Single-patch trial hit rates (circles) and false alarm rates (squares) versus without SC optogenetic stimulation for the four mice, plotted separately for contralateral (orange) and ipsilateral (blue) blocks. Open symbols are data from individual mice. Error bars indicate $95 \% \mathrm{Cl}$. Ellipses are $95 \%$ Cls of the population mean. $\boldsymbol{C}$, Same as $\boldsymbol{B}$, but for double-patch trials. $\boldsymbol{D}$, Reaction times versus without $\mathrm{SC}$ optogenetic stimulation for single-patch trials. Open symbols are median reaction time from individual mice, error bars are $95 \%$ Cls. Ellipses are $95 \%$ Cls of the population mean. $\boldsymbol{E}$, Same as $\boldsymbol{D}$, but for double-patch trials.

contralateral visual stimulus but generally $<100 \%$, and were centered in the intermediate layers but included spread into the more superficial and deep layers.

We first assessed whether the SC in mice was involved in the orientation change detection by applying SC inhibition during a 300 -ms interval spanning the time from the visual change to the onset of the response window (Fig. $3 A$ ). Without SC inhibition, a cohort of four mice achieved high levels of performance for detecting $15^{\circ}$ orientation changes (average hit rate of $90.3 \%$ and average false alarm rate of $10.2 \%$ ). SC inhibition during the 300 $\mathrm{ms}$ interval significantly reduced hit rate specifically for orientation changes in the visual hemifield contralateral to the site of SC inhibition. Reductions were evident in both single-patch trials (Fig. 3B), where contralateral hit rates with inhibition were all substantially below unity line (without inhibition: $86.9 \pm 6.6 \%$, mean $\pm 95 \% \quad C I$; with inhibition: $64.7 \pm 13.0 \%, p=0.057$, Wilcoxon rank sum test for the population; four of four individual mice had significant reductions in hit rates with SC inhibition by $\chi^{2}$ test, $p<0.05$ ) and double-patch trials (Fig. 3C; without inhibition: $91.7 \pm 4.1 \%$; with inhibition: $60.2 \pm 12.2 \%, p=0.029$; four of four mice significant by $\chi^{2}$ test, $p<0.0001$ ). In contrast to these strong contralateral effects, the detection of orientation changes in the ipsilateral visual hemifield was unchanged by unilateral SC inhibition for both single-patch trials (Fig. $3 B ; p=0.57$, Wilcoxon rank sum test) and double-patch trials (Fig. $3 C$, $p=0.99$ ), consistent with the retinotopic organization of the SC. The false alarm rates were not markedly changed by SC inhibition for either the contralateral or ipsilateral trials, as evident in Figure 3, $B$ and $C$ (contralateral single-patch: $p=0.99$, Wilcoxon rank sum test; double-patch: $p=0.31$; ipsilateral single-patch: $p=0.89$; double-patch: $p=0.89$ ). SC inhibition also significantly increased reaction times for detecting contralateral orientation changes, as evident in the results from the doublepatch trials shown in Figure $3 E$ (without inhibition: $404.1 \pm$ $32.4 \mathrm{~ms}$; with inhibition: $462.8 \pm 39.3 \mathrm{~ms} ; p=0.11$, Wilcoxon rank sum test for the population; four of four mice had significant increases in reaction time by SC inhibition with $p<0.01$ in rank sum test), and less obviously for single-patch trials (Fig. $3 D$; without inhibition: $419.1 \pm 54.0 \mathrm{~ms}$; with inhibition: $462.2 \pm 40.9 \mathrm{~ms} ; p=0.31$; three of four mice had significant increases in reaction time with $p<0.01$ for rank sum test). Reaction times for ipsilateral orientation changes were not altered by SC inhibition for either single-patch or doublepatch trials, as shown in Figure 3, $D$ and $E$ (single-patch: $p=0.89$; double-patch: $p=0.89$ ). These spatially specific deficits in response accuracy and reaction time demonstrate that SC inhibition during this 300-ms window disrupts choice behavior but do not identify which stage of processing (i.e., anticipation, visual processing, or response preparation) was most strongly affected.

\section{Time course of firing rate modulation during the task}

To identify which aspects of task performance were affected, we tested the effects of SC inhibition applied within more specific time windows. Before these experiments, to identify appropriate time windows, we first documented when SC neurons were modulated during the task (Fig. 4). Electrophysiological recordings in two mice well trained on the task revealed that neurons in the SC exhibited a phasic response after the Gabor patch in the contralateral visual field changed its orientation (Fig. 4A). The detailed patterns of evoked spikes varied across individual neurons, but the overall timing of the firing rate modulation was similar throughout the $1.5 \mathrm{~mm}$ depth of the SC (Fig. 4B). A summary of the firing rate pattern (Fig. $4 C$ ) across the total of 260 neurons shows a phasic visual response $\sim 50-100 \mathrm{~ms}$ after the orientation change, followed by a gradual drop in activity over the subsequent $100 \mathrm{~ms}$ (Fig. 4C); by the time of lick onset (Fig. $4 C$, inset, distribution), firing rate was lower than the baseline before the orientation change. Overall, $43 \%$ of the neurons (112 of 260) exhibited a significant increase in activity $50-150 \mathrm{~ms}$ after the orientation change ( $p<0.05$, Wilcoxon rank sum test; comparison with activity $0-100 \mathrm{~ms}$ before the orientation change). These data demonstrate that SC neurons were robustly modulated by the behaviorally relevant stimulus event in our task (near-threshold orientation change), but overall did not exhibit much activity immediately before or during the lick response itself.

\section{Temporal precision of SC inhibition on detection performance}

Based on the firing rate modulation during the task, we defined three nonoverlapping 100-ms temporal epochs (Fig. 4C). "Epoch 1 " (from -50 to $50 \mathrm{~ms}$ with respect to the orientation change) corresponded to the prechange baseline of SC activity; a behavioral effect caused by inhibition during this epoch might suggest the involvement of SC activity in anticipation during the task. "Epoch 2" (from 50 to $150 \mathrm{~ms}$ after the orientation change) overlapped with the phasic visual response of SC neurons to the orientation change; a behavioral effect here would indicate the importance of SC visual processing for the perceptual decision. 

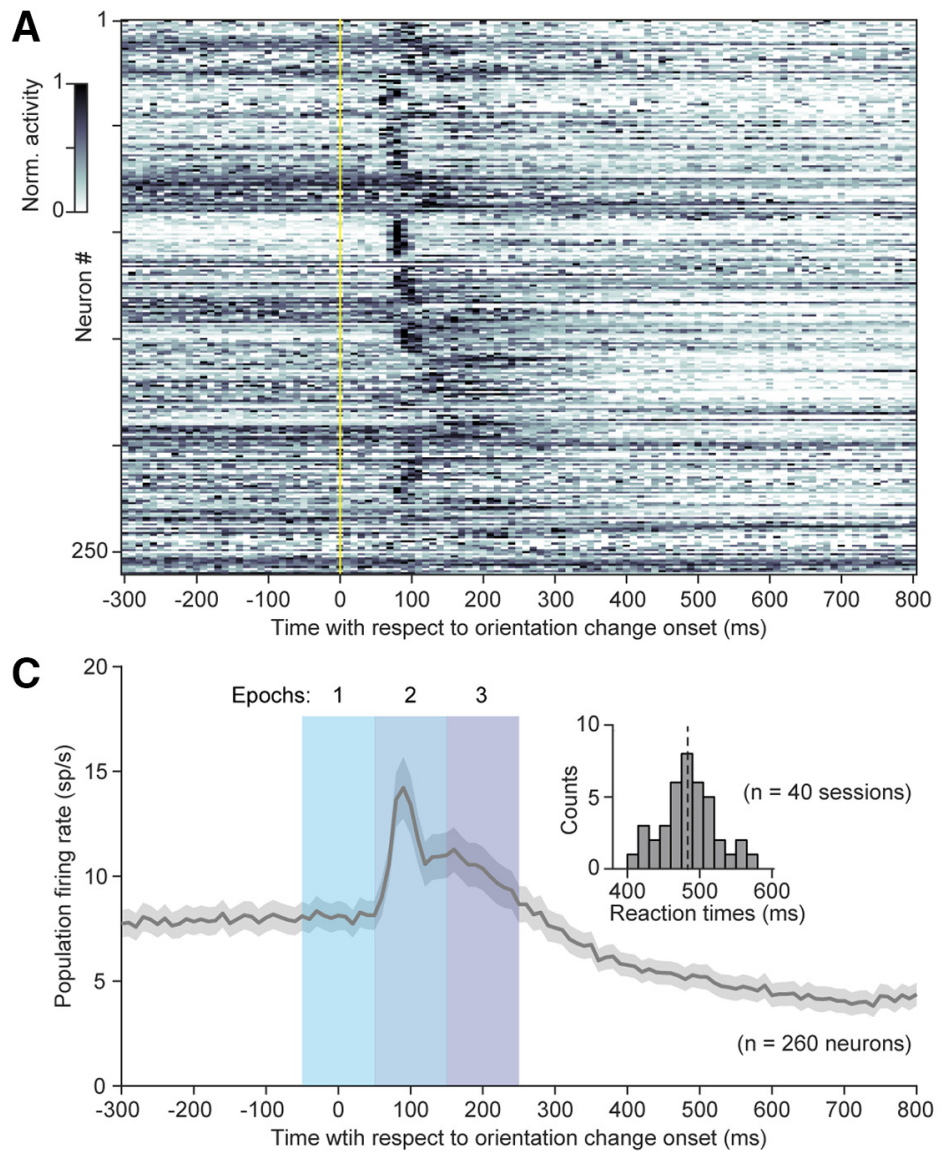

Figure 4. Firing rate modulation of $S C$ neurons during the detection task. $A$, Normalized spike rates of all recorded SC neurons from two mice aligned on the onset of contralateral orientation changes $\left(12^{\circ}\right)$. Each row shows the normalized spike counts in 10-ms bins from an individual neuron. Neurons $(n=260)$ are sorted by recording depth from the most superficial (top) to the deepest (bottom). $\boldsymbol{B}$, SC depth for each of the neurons, following the same sorting convention as in $\boldsymbol{A}$. C, Mean firing rate across all SC neurons. Solid line indicates the mean, and shaded region indicates \pm SEM. Vertical blue shaded regions indicate the three temporal epochs chosen for the SC inhibition experiment. Inset, Distribution of reaction times across all recording sessions; dashed line indicates the median.

"Epoch 3" (from 150 to $250 \mathrm{~ms}$ after change onset) corresponded to the period of declining firing rate after the initial visual burst; a behavioral effect caused by SC inhibition during this epoch might indicate a role for the SC in response preparation or later stages of visual processing.

SC inhibition during epoch 1 (Fig. 5A) had little effect on task performance. Without SC inhibition, mice detected $12^{\circ}$ orientation changes with average hit rates of $87.5 \%$ and false alarm rates of $12.8 \%$. These rates were similar during epoch 1 inhibition for contralateral orientation changes, for both single-patch (Fig. $5 B$; $p=0.74$, Wilcoxon signed-rank test, comparing population hit rate with and without inhibition) and double-patch trials (Fig. $5 C ; p=0.15)$. Similarly, hit rates for ipsilateral orientation changes were also not altered by epoch 1 SC inhibition, as shown in Figure 5, $B$ and $C$ (single-patch: $p=0.31$; double-patch: $p=0.84$ ). Epoch 1 inhibition had variable effects on false alarm rates. It did not significantly alter false alarm rates during contralateral blocks for either single-patch trials (Fig. $5 B ; p=0.25$, Wilcoxon signed-rank test) or double-patch trials (Fig. $5 C$; $p=0.11$ ), but induced a small but significant increase of false alarm rates in both single-patch trials (Fig. $5 B ; p=0.035$ ) and double-patch trials (Fig. $5 C ; p=0.016$ ) during ipsilateral blocks. Similarly, there were no significant effects of SC inhibition on reaction times for either contralateral (Fig. 5D; single-patch, $p=0.84$, Wilcoxon signed-rank test; Fig. 5E: double patch,

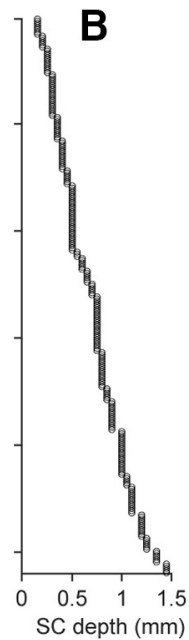

$p=0.055)$ or ipsilateral orientation changes (Fig. 5D: single patch, $p=0.38$; Fig. $5 E$ : double patch, $p=0.11)$. The absence of changes in hit rate or reaction times by SC inhibition during this epoch suggests that SC activity during the delay period (as the mice might have been anticipating the orientation change) was not essential for task performance.

In contrast, inhibiting SC visual activity during epoch 2 (Fig. $5 F$ ) caused profound deficits on detection performance. Epoch 2 inhibition significantly reduced hit rates for contralateral orientation changes in both single-patch trials (Fig. $5 G, p=0.0078$, Wilcoxon signed-rank test comparing epoch 2 SC inhibition and no inhibition) and double-patch trials (Fig. $5 H, p=0.0078)$. SC inhibition during epoch 2 did not have a significant effect on hit rates for ipsilateral orientation changes in either single-patch trials (Fig. $5 G, p=0.055$ ) or double-patch trials (Fig. $5 H, p=0.55)$, consistent with the retinotopic organization of the SC. SC inhibition during this epoch also tended to increase false alarm rates for both contralateral (Fig. 5G: single patch: $p=0.20$, Wilcoxon signed-rank test; Fig. $5 \mathrm{H}$ : double patch: $p=0.03$ ) and ipsilateral (single patch: $p=0.045$; double patch: $p=0.078$ ) orientation changes. Consequently, sensitivity and response criterion also significantly changed with inhibition during this epoch. Sensitivity was significantly reduced with SC inhibition for detecting contralateral orientation changes (single patch: without inhibition, $2.12 \pm 0.29$; with inhibition: $1.31 \pm 0.24 ; p=0.0078$, Wilcoxon signed-rank test; double patch: without inhibition, $2.13 \pm 0.31$; with inhibition, $1.01 \pm 0.28$; $p=0.0078$ ) but not the ipsilateral ones (single patch: without inhibition, $2.61 \pm 0.24$; with inhibition, $2.41 \pm 0.50 ; p=0.38$; double patch: without inhibition, $2.61 \pm 0.31$; with inhibition, $2.39 \pm 0.52$; $p=0.38$ ). SC inhibition caused an increase in response criterion for contralateral changes (single patch: without inhibition, $0.06 \pm 0.17$; with inhibition, $0.25 \pm 0.28 ; p=0.11$; double patch: without inhibition, $-0.08 \pm 0.10$; with inhibition, $0.17 \pm 0.24 ; p=0.02)$ and a reduction for ipsilateral changes (single-patch: without inhibition, $0.06 \pm 0.19$; with inhibition, $-0.25 \pm 0.15 ; p=0.02$; doublepatch: without inhibition, $-0.09 \pm 0.16$; with inhibition, -0.31 $\pm 0.18 ; p=0.07$ ); the more lenient criterion was presumably an adaptation to the disruption of visual processing.

Inhibiting SC visual activity during epoch 2 also delayed the timing of detection for contralateral visual changes. Reaction times for contralateral orientation changes with epoch 2 SC inhibition were significantly longer than those without SC inhibition (Fig. 5I, single patch: $p=0.016$, Wilcoxon signed-rank test; Fig. 5J, double patch: $p=0.0078$ ). For ipsilateral orientation changes, the effects were more variable: SC inhibition did not alter reaction times for single-patch trials (Fig. 5I, $p=0.99$ ), but caused a small reduction for double-patch trials (Fig. 5J, $p=0.039$ ). Thus, 

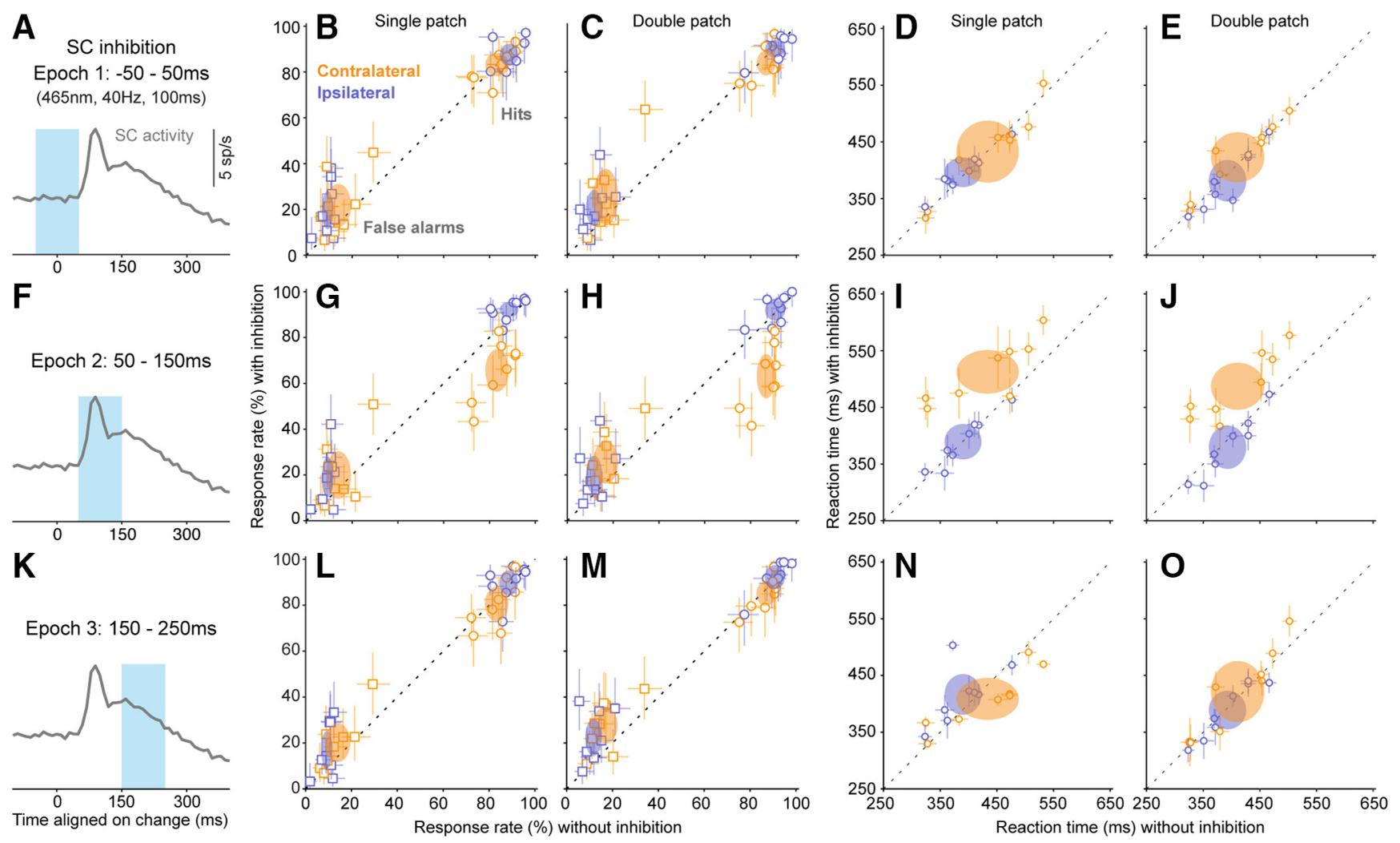

Figure 5. The effect of $\mathrm{SC}$ inhibition on detection performance and reaction times had remarkable temporal specificity. $A$, For epoch 1, optogenetic inhibition of the $\mathrm{SC}(465 \mathrm{~nm}, 40 \mathrm{~Hz})$ was applied from -50 to $50 \mathrm{~ms}$ with respect to the orientation change. SC activity trace is the same data as shown in Fig. 4C. B, Single-patch trial hit rates (circles) and false alarm rates (squares) versus without $\mathrm{SC}$ inhibition applied during epoch 1 for eight mice, plotted separately for contralateral (orange) and ipsilateral (blue) blocks. Open symbols are mean response rates from individual mice; error bars are $95 \%$ Cls. Ellipses are $95 \%$ Cls of the population mean. C, Same as $\boldsymbol{B}$, but for double-patch trials. $\boldsymbol{D}$, Reaction times versus without SC inhibition during epoch 1 for single-patch trials. $\boldsymbol{E}$, Reaction times versus without $\mathrm{SC}$ inhibition during epoch 1 for double-patch trials. $\boldsymbol{F}$, For epoch 2, optogenetic inhibition of the $S C$ was applied from 50 to $150 \mathrm{~ms}$ with respect to the orientation change. $\mathbf{G}$, Same as $\boldsymbol{B}$, except that $S \mathbf{C}$ inhibition was applied during epoch 2 . $\boldsymbol{H}$, Same as $\boldsymbol{G}$, but for double-patch trials. $\boldsymbol{I}$, Reaction times versus without $\mathbf{S C}$ inhibition during epoch 2 for single-patch trials. J, Reaction times versus without SC inhibition during epoch 2 for double-patch trials. $\boldsymbol{K}$, For epoch 3, optogenetic inhibition of the SC was applied from 150 to 250 ms with respect to the orientation change. $\boldsymbol{L}$, Same as $\boldsymbol{B}$, except that SC inhibition was applied during epoch 3 . $\boldsymbol{M}$, Same as $\boldsymbol{L}$, but for double-patch trials. $\boldsymbol{N}$, Reaction times versus without $S C$ inhibition during epoch 3 for single-patch trials. $\boldsymbol{O}$, Reaction times versus without $S C$ inhibition during epoch 3 for double-patch trials. Overall, changes in hit rates and reaction times were only evident with $\mathrm{SC}$ inhibition when applied during epoch 2 for contralateral orientation changes.

epoch 2 SC inhibition during double-patch trials caused opposite effects on reaction time between contralateral and ipsilateral orientation changes $(p=0.038$, two-way ANOVA), suggesting a cross-hemifield interaction in the presence of a visual distractor. Together, these effects on hit rate and reaction times demonstrate that the early visual responses of SC neurons to the orientation change were crucial for the ability of mice to perform this visual change detection task.

Finally, SC inhibition during epoch 3 (Fig. 5K) had little effect on task performance. Hit rates were not significantly changed by SC inhibition during epoch 3 for either contralateral (Fig. $5 L, M$; single patch: $p=0.38$, Wilcoxon signed-rank test; double patch: $p=0.20$ ) or ipsilateral orientation changes (single patch: $p=0.84$; double patch: $p=0.47$ ). Epoch 3 SC inhibition caused small but significant increases in false alarm rates for both contralateral blocks (single-patch: $p=0.016$; double-patch: $p=0.023$ ) and ipsilateral blocks (single-patch: $p=0.11$; double-patch: $p=0.0078$ ). Reaction times were also not significantly affected, for either contralateral changes (Fig. $5 \mathrm{~N}$, single patch: $p=0.078$; Fig. $5 \mathrm{O}$, double patch: $p=0.38$ ) or ipsilateral changes (single patch: $p=0.055$; double patch: $p=0.55$ ). The absence of effects on hit rates during this epoch suggests that this later stage of evoked SC activity was not essential for task performance and underscores the importance of the phasic visual response that immediately precedes this epoch.
In summary, we found that the most consistent effects of inhibiting SC activity on hit rates and reaction times were specific to the 100-ms time epoch that matched visual-evoked phasic activity in the SC. These results indicate that SC visual responses are crucial for the ability of mice to detect the orientation changes in our task.

\section{Involvement of the mouse SC in visual selection revealed by psychometric curves}

In the results presented so far, we showed that the ability to detect a $12^{\circ}$ orientation change was markedly impaired when inhibition was applied during the initial SC visual response. There were also slightly larger effects on double-patch trials than on single-patch trials, which might be expected given the widespread evidence in other species that the SC is involved in visual target selection (Mysore and Knudsen, 2011; Krauzlis et al., 2013). To test this further, we next investigated SC inhibition using a range of stimulus values and constructed psychometric curves. For these experiments, SC inhibition was applied only during epoch 2.

SC inhibition caused systematic changes in psychometric curves for contralateral orientation changes that were larger in the double-patch than in the single-patch condition. As illustrated by single-patch data from sample mouse 1 (Fig. 6A), 

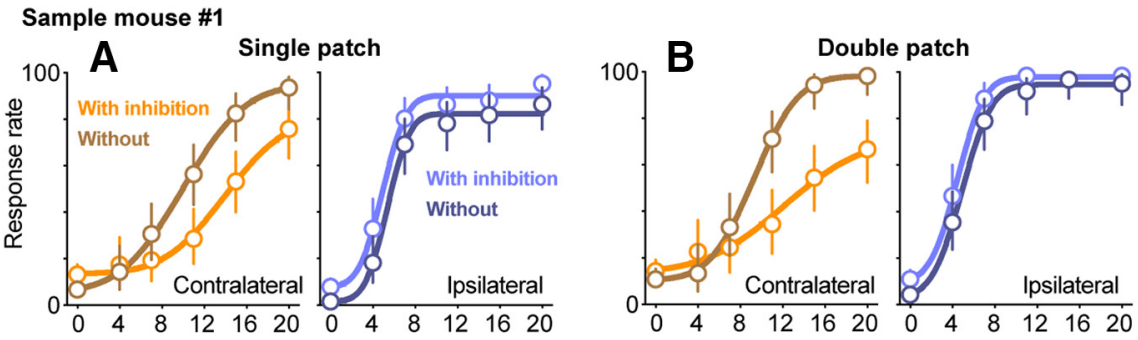

Population $(n=8)$
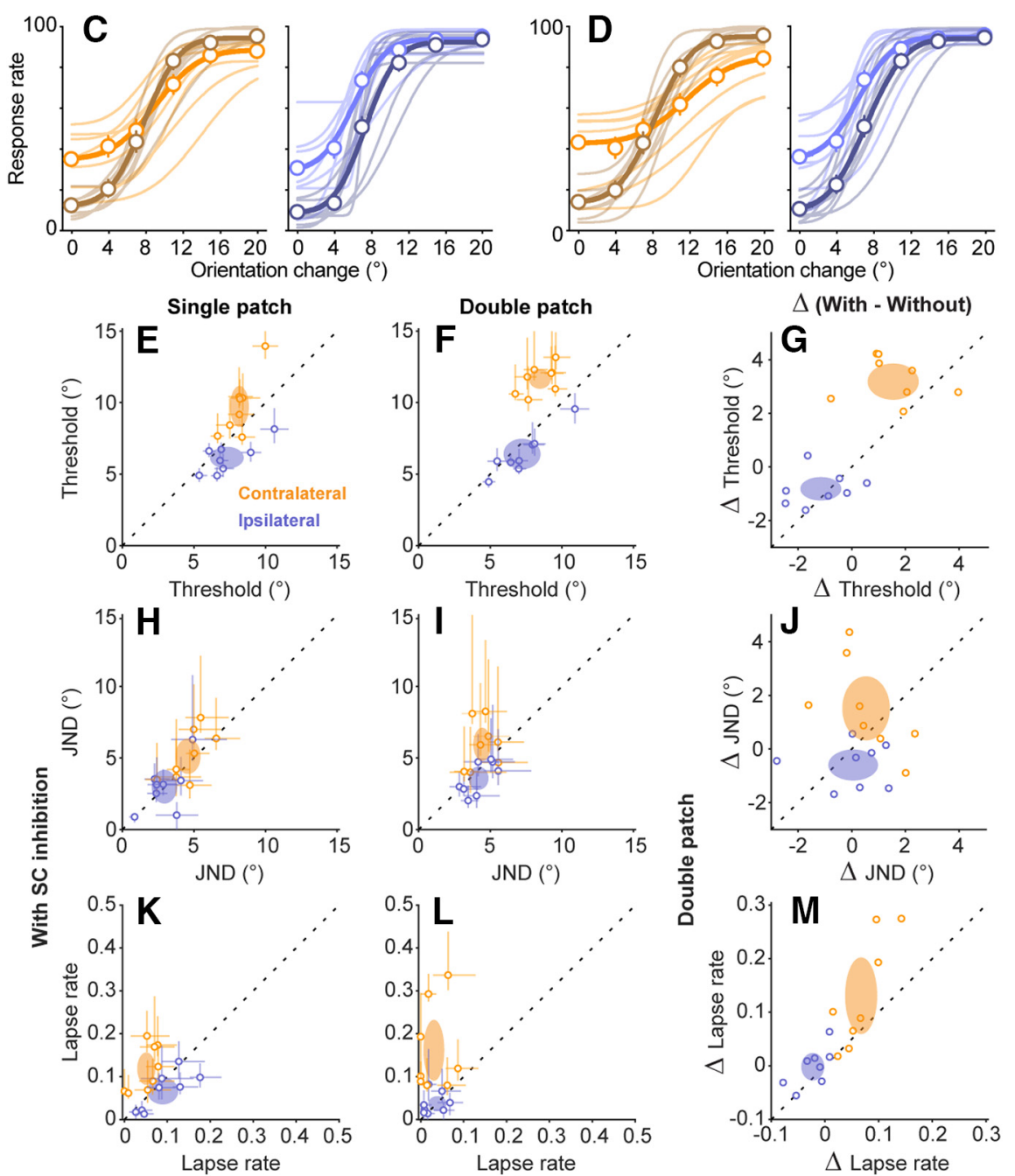
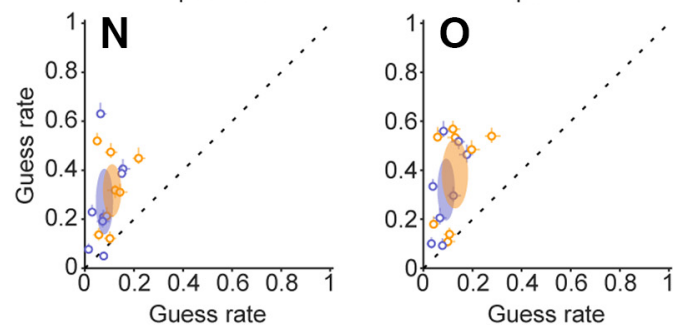

Without SC inhibition

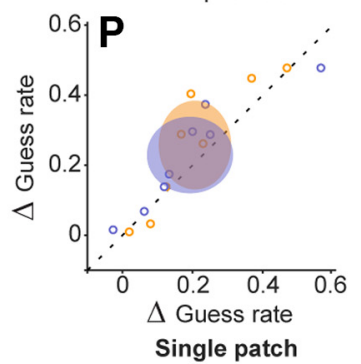

Figure 6. Effects of $S C$ inhibition on psychometric curves. $A, B$, Psychometric data and curves from a sample mouse with and without SC inhibition applied during epoch 2 for single-patch $(\boldsymbol{A})$ and double-patch $(\boldsymbol{B})$ trials. Open symbols are the mean response rates from individual mice, and error bars indicate $95 \%$ Cls. Each value is based on 52-69 trials for conditions with orientation change, or 266-329 trials with $0^{\circ}$ orientation change. Curves show fitted cumulative Gaussian functions. SC inhibition shifted psychometric curves downward for contralateral orientation changes. C, D, Psychometric curves across all mice $(n=8)$ with and without SC inhibition for contralateral (orange) and ipsilateral (blue) orientation changes. Thin lines are fitted curves from individual mice, and thick lines show population average, for single-patch $(\boldsymbol{C})$ and double-patch $(\boldsymbol{D})$ trials. Error bars again indicate $95 \% \mathrm{Cls}$, and each value is based on $372-446$ trials, or $1894-2185$ trials $\left(0^{\circ}\right.$ orientation without SC inhibition (dark orange) the response rate on contralateral trials went from $\sim 10 \%$ to $>95 \%$ as the orientation change was increased from $4^{\circ}$ to $20^{\circ}$. With SC inhibition (light orange), the curve was shifted rightward and/or downward, reaching an asymptote of only $75 \%$. These effects of SC inhibition were more pronounced in double-patch trials (Fig. $6 B)$, as follows: the curve with SC inhibition was shallower and reached a lower asymptote of $\sim 65 \%$. Thus, the detection deficits caused by SC inhibition appeared to be worse when a competing visual stimulus was present in the other hemifield. Similar effects were found across all the mice (Fig. $6 C, D$ ), albeit with some variability across animals, and an overall tendency for SC inhibition to increase false alarms (i.e., responses for $0^{\circ}$ orientation change). Specifically, the flattening of the psychometric curves caused by SC inhibition was more pronounced in the double-patch trials (Fig. $6 D$, orange curves) than in the single-patch trials (Fig. 6C).

To quantify how SC inhibition affected psychometric performance and to identify whether those effects might depend on the presence of competing visual stimuli, we extracted four independent parameters of the cumulative Gaussian curves fitted to the data from individual mice, following methods described previously (Wang and Krauzlis, 2018). These four parameters were as follows: (1) detection threshold, defined as the mean of the fitted Gaussian function; (2) JND, the SD of the fitted Gaussian function multiplied by $\sqrt{ } 2$, which is the minimum change in the visual orientation that would result in detection at least $50 \%$ of the time; (3) lapse rate, the upper asymptote of the cumulative function, and often attributed to nonsensory perceptual errors; and (4) guess rate, defined as the lower asymptote of the fitted function, and related to the subject's response criterion or nonperceptual motor bias.

SC inhibition increased detection thresholds for contralateral orientation changes

\footnotetext{
$\leftarrow$

change), pooled across mice. $\boldsymbol{E}, \boldsymbol{F}$, Comparison of detection thresholds versus without $S C$ inhibition for contralateral (orange) and ipsilateral (blue) orientation changes, for singlepatch $(\boldsymbol{E})$ and double-patch $(\boldsymbol{F})$ trials. Open symbols are median thresholds from individual mice, and error bars are $95 \%$ Cls. Ellipses are $95 \%$ Cls of population mean. $\mathbf{G}$, Comparison of the changes in thresholds ( $\Delta$ threshold) induced by $S C$ inhibition between single-patch ( $x$-axis) and double-patch ( $y$ axis) trials. $\boldsymbol{H}-\boldsymbol{J}$, JNDs with and without $S C$ inhibition. $\boldsymbol{K}-\boldsymbol{M}$, Lapse rates with and without $S C$ inhibition. $\boldsymbol{N}-\boldsymbol{P}$, Guess rates with and without $S C$ inhibition. Other conventions are as above.
} 


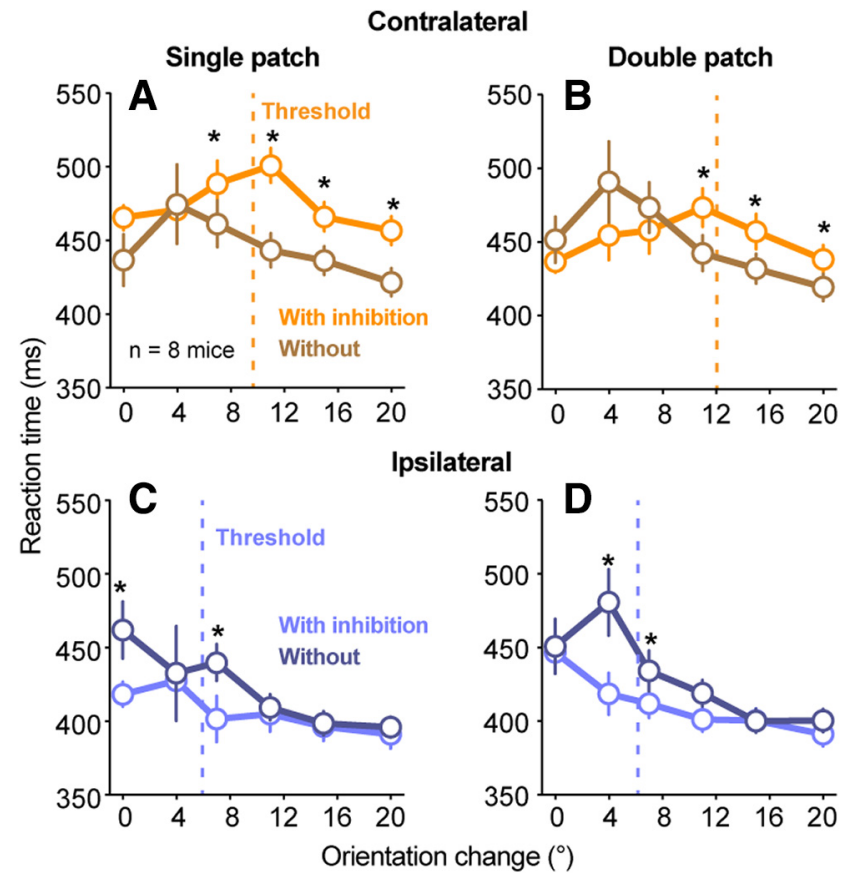

Figure 7. Effects of $\mathrm{SC}$ inhibition on reaction times for psychometric data. $\boldsymbol{A}, \boldsymbol{B}$, Population reaction times ( $n=8$ mice) plotted as a function of orientation change amplitude with (orange) and without (brown) SC inhibition for single-patch $(\boldsymbol{A})$ and double-patch $(\boldsymbol{B})$ trials. SC inhibition increased contralateral reaction times for orientation changes that were larger than the average threshold for detection (dashed line: population mean threshold with SC inhibition across mice). Symbols are median values, and error bars indicate $95 \% \mathrm{Cls}$. *Significant difference ( $p<0.05$, Wilcoxon signed-rank test) in reaction time with and without $S C$ inhibition at that particular orientation change amplitude. $\boldsymbol{C}, \boldsymbol{D}$, Same as $\boldsymbol{A}$ and $\boldsymbol{B}$, but for ipsilateral orientation changes. SC inhibition decreased ipsilateral reaction times for orientation change amplitudes near threshold.

(Fig. 6E,F). These increases were significant for both single-patch trials (Fig. $6 E, p=0.016$, Wilcoxon signed-rank test) and doublepatch trials (Fig. 6F, $p=0.0078$ ), and the effects on detection threshold were significantly larger for double-patch trials (Fig. $6 G, 3.25 \pm 0.59^{\circ}$, mean $\pm 95 \% \mathrm{CI}$ ) than for single-patch trials $\left(1.55 \pm 0.95^{\circ} ; p=0.039\right.$, Wilcoxon signed-rank test). Thus, SC inhibition decreased the likelihood of detecting smaller contralateral orientation changes, and this effect was larger when a competing stimulus was present in the ipsilateral hemifield. For ipsilateral orientation changes, SC inhibition caused small decreases in detection thresholds during both single-patch trials ( $p=0.039$, Wilcoxon signed-rank test) and double-patch trials $(p=0.016)$ that did not differ in magnitude (Fig. 6G, $p=0.55$ ).

SC inhibition caused subtle changes in JND. For contralateral orientation changes, SC inhibition did not significantly alter JND during single-patch trials (Fig. $6 H, p=0.25$, Wilcoxon signedrank test) but caused a small increase in JND during double-patch trials (Fig. 6I, $p=0.042$ ). This result suggests that suppressing the visual activity of SC neurons decreased the perceptual sensitivity for mice in our task, at least when a competing stimulus was present. However, the magnitude of contralateral JND increase caused by SC inhibition did not significantly differ between single-patch and double-patch trials (Fig. $6 J, p=0.38$ ). For ipsilateral orientation changes, SC inhibition did not significantly alter JND for either single-patch trials (Fig. $6 H, p=0.46$ ) or double-patch trials (Fig. 6I, $p=0.11$ ).

SC inhibition caused a spatially specific increase in lapse rates. For contralateral orientation changes, SC inhibition significantly increased lapse rates in both single-patch trials (Fig. $6 K$, $p=0.0078$, Wilcoxon signed-rank test) and double-patch trials (Fig. $6 L, p=0.0078$ ), and the size of the increase in lapse rates caused by SC inhibition in double-patch trials was greater than that in single-patch trials (Fig. $6 M, p=0.039$, Wilcoxon signedrank test). Thus, the increased lapse rate caused by SC inhibition was amplified in the presence of a distractor in the ipsilateral hemifield, consistent with the idea that lapse rate is linked to uncertainty during the perceptual choice (Pisupati et al., 2019). These effects were also spatially specific, because lapse rates for detecting ipsilateral orientation changes were not significantly altered by SC inhibition for either single-patch (Fig. $6 K, p=0.074$ ) or double-patch (Fig. $6 L, p=0.90$ ).

Finally, SC inhibition caused nonspecific increases in guess rates. For single-patch trials, SC inhibition increased guess rates for detecting both contralateral (Fig. $6 N, p=0.0078$, Wilcoxon signed-rank test) and ipsilateral $(p=0.016)$ orientation changes; the increases in guess rates for contralateral and ipsilateral changes did not differ significantly ( $p=0.88$, two-way ANOVA with conditions of spatial locations and SC inhibition). Similarly, during double-patch trials, SC inhibition also increased guess rates for both contralateral detections (Fig. 6O, $p=0.0078$ ) and ipsilateral detections $(p=0.0078)$, also without spatial specificity $(p=0.78$, two-way ANOVA). For both contralateral and ipsilateral trials, the increased guess rates caused by SC inhibition did not differ between single-patch and double-patch conditions (Fig. $6 P$, contralateral: $p=0.15$, Wilcoxon signed-rank test, ipsilateral: $p=0.11$ ). These spatially nonspecific increases of guess rates, along with the nonspecific increase in false alarm rates when we inhibited SC activity during three temporal epochs (Fig. 5), are consistent with changes in response bias on trials with SC inhibition. Similar but larger increases in false alarm rates with SC inhibition have been found in mice performing visual go/no-go tasks with suprathreshold orientation stimuli $(\mathrm{Hu}$ et al., 2019).

Inhibiting SC visual activity also significantly altered reaction times, but mostly for contralateral changes that were large enough to be detected (Fig. 7). For contralateral orientation changes, SC inhibition increased reaction times for near-threshold and superthreshold orientation changes, but not for smaller orientation changes (Fig. 7A,B). For ipsilateral orientation changes, the effect of SC inhibition on reaction times was smaller and more variable (Fig. 7C,D).

In summary, results from our psychometric analysis revealed that inhibiting SC visual activity for $100 \mathrm{~ms}$ caused a spatially selective deficit in several aspects of detection performance, including increases in detection thresholds and lapse rates for visual events represented by the affected SC neurons. The magnitude of these effects was larger in the presence of a competing visual distractor, consistent with a role of the SC in visual target selection as well as visual processing. In addition to deficits in the contralateral hemifield, inhibiting SC visual activity also caused a complementary improvement for some aspects of ipsilateral detection, including decreases in detection thresholds and decreases in reaction time, again consistent with a role for the SC in mediating competitive interactions across the visual field.

\section{Detection deficits not caused by retinal stimulation artifacts}

One concern with optogenetic manipulations in mice is that scattered light from the optic probe might alter visual performance by activating the retina from inside the head of the mouse, rather than by changing the activity of neurons targeted for opsin expression. To assess whether the detection deficits we observed 
A
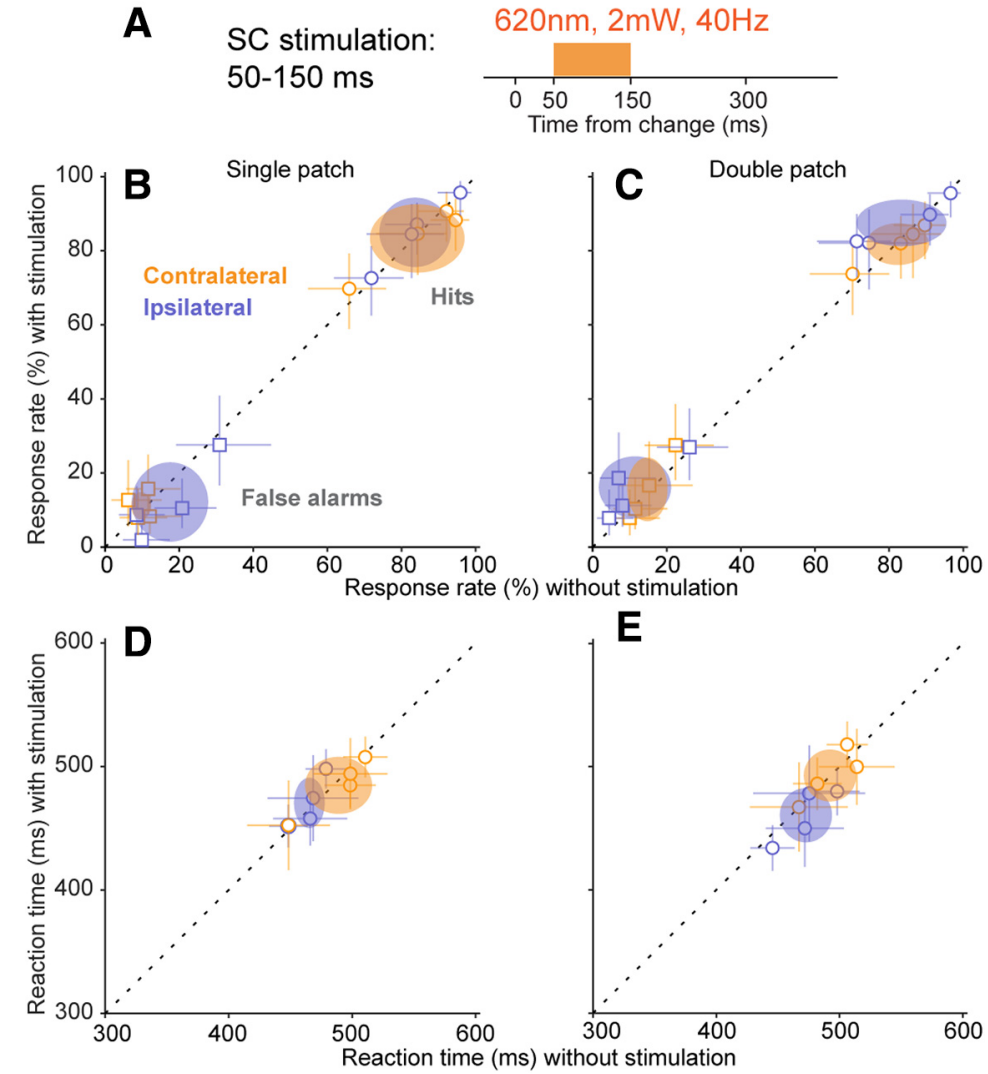

Figure 8. Orange light stimulation in the $\mathrm{SC}$ did not alter detection performance. $\boldsymbol{A}$, Timing of the control $40 \mathrm{~Hz}$ orange light ( $620 \mathrm{~nm}$ wavelength) stimulation applied in the SC during epoch 2, aligned to the orientation change. $\boldsymbol{B}_{\boldsymbol{B}}$ Single-patch trial hit rates (circles) and false alarm rates (squares) versus without orange light stimulation, plotted separately for contralateral (orange) and ipsilateral (blue) blocks. Conventions are the same as in Figure 3. C, Same as B, but for double-patch trials. $\boldsymbol{D}$, Reaction times versus without orange light stimulation for single-patch trials. Open symbols are the median reaction time from individual mice, and error bars are $95 \%$ Cls. Ellipses are $95 \%$ Cls of population mean. $\boldsymbol{E}$, Same as $\boldsymbol{D}$, but for double-patch trials.

with our optogenetic manipulation might be due to this type of nonspecific retinal activation, we performed a set of control experiments in a subset of Vgat-Cre mice used for data in Figures 2-6. For these controls (Fig. 8A), we again applied light within the SC during epoch 2 using a $12^{\circ}$ orientation change, but instead of blue light ( $465 \mathrm{~nm}$ wavelength) we used orange light (620 nm wavelength). Orange light provides a strong test for possible retinal artifacts: the longer wavelength spreads further than blue light in brain tissue but does not activate ChR2 (Danskin et al., 2015).

The orange light manipulation had no effect on task performance. For single-patch conditions, as shown in Figure 8B, orange light caused no significant changes in hit rates for either contralateral trials ( $p=0.88$, Wilcoxon signed-rank test) or ipsilateral trials $(p=0.15)$. Similarly, orange light did not alter false alarm rates (contralateral: $p=0.57$; ipsilateral: $p=0.11$ ). For double-patch conditions, as shown in Figure $8 C$, orange light again did not change hit rates (contralateral: $p=0.72$; ipsilateral: $p=0.28$ ) or false alarm rates (contralateral: $p=0.65$; ipsilateral: $p=0.14$ ). Reaction times were also unaffected for both contralateral orientation changes (Fig. $8 D$, single-patch: $p=0.38$; Fig. $8 E$, double-patch: $p=0.88$ ) and ipsilateral orientation changes (Fig. $8 D$, single-patch: $p=0.38$; Fig. $8 E$, double-patch: $p=0.25$ ). These results rule out the possibility that the deficits in task performance we observed during optogenetic stimulation were caused by artifactual stimulation of the retina.

\section{Detection deficits is not related to saccades}

Another concern is that optogenetic inhibition in the SC might induce saccades (Wang et al., 2015), altering performance in the task by displacing the retinal image rather than by changing visual processing. To assess this possibility, we recorded the position of eye contralateral to the SC inhibition side during the visual epoch experiment (Fig. 5) in a subset of mice and measured the probability of saccades with and without SC inhibition. Since detection deficits were specific to SC inhibition during epoch 2, we focused our analysis on that experimental condition.

Mice made very few saccades during our task, regardless of SC inhibition. As shown by a sample trial with saccades (Fig. 9A), when saccades did occur, they were readily detected and tended to have larger horizontal than vertical components. Overall, the probability of saccades was $<3 \%$ throughout the trial, regardless of SC inhibition (Fig. 9B), although SC inhibition did produce a slight increase in saccade probability (Fig. 9B, inset). To quantify this pattern, we compared saccade probability with and without epoch 2 inhibition during the time window from 50 to $800 \mathrm{~ms}$ with respect to the orientation change. In single-patch trials, SC inhibition did not change saccade probability on either change trials $(p=0.44$, Wilcoxon signedrank test; Fig. $9 C$ ) or no-change trials $(p=0.31)$. In double-patch trials, SC inhibition caused a small but not significant increase in saccade probability for both change trials $(p=0.063)$ and no-change trials $(p=0.063)$. Thus, the SC inhibition generally did not have significant effects on saccade probability, and the change in the probability of saccades was much smaller than the effect of SC inhibition on hit rates (Fig. 5). We conclude that the effects of SC inhibition on task performance were not an artifact of evoked saccades.

\section{Discussion}

Our findings highlight the importance of the SC in mouse visual perception. We found that the mouse SC is necessary for detection behavior during a visual orientation change task. The most important temporal epoch was a 100-ms window that overlapped with the visual response of SC neurons. SC inhibition during this epoch caused profound deficits in detecting contralateral visual changes, including reduced perceptual sensitivity, increased detection threshold, prolonged reaction times, and increased nonsensory lapse rates. In addition, deficits in visual detection were larger in the presence of a competing stimulus, consistent with the known role for the SC in visual selection.

\section{Superior colliculus and early visual processing in mice}

The retinocollicular pathway is the dominant visual pathway in mice. More than $85 \%$ of RGCs in mice project to the superficial layers of the SC (Hofbauer and Dräger, 1985; Ellis et al., 2016), most RGCs that project to the lateral geniculate nucleus also 

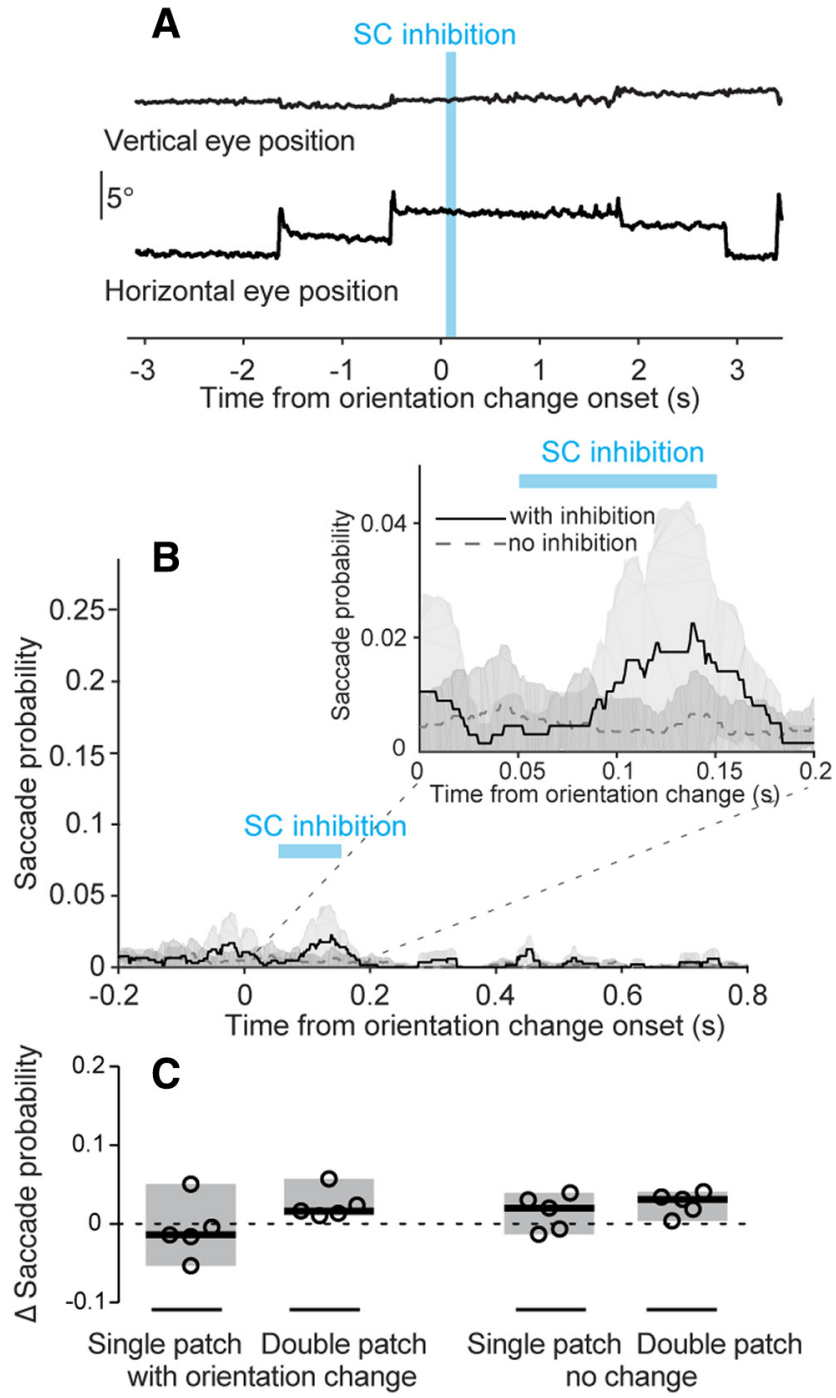

Figure 9. SC inhibition did not significantly alter saccade probability. $\boldsymbol{A}$, Vertical and horizontal eye position traces from an example trial with $\mathrm{SC}$ inhibition applied during epoch 2 (blue bar). The orientation change occurred on the same side as the tracked eye. $\boldsymbol{B}$, Saccade probability within each 1-ms time bin during double-patch trials with (solid black) and without (dashed gray) SC inhibition. The orientation change was again on the same side as the tracked eye and contralateral to the side with SC inhibition ( $n=5$ mice). Timing of the SC inhibition indicated by the blue bar. Inset shows an expanded view of saccade probability for the temporal interval between 0 and $200 \mathrm{~ms}$ after the orientation change. C, Change in saccade probability (with SC inhibition minus no SC inhibition) during a 750-ms interval (from 50 to $800 \mathrm{~ms}$ after the onset of the change epoch). Showing four different trial types within contralateral cue blocks. Open symbols are mean change in saccade probability from individual mice. Black lines indicate population medians. Shaded regions indicate 95\% Cls.

project to the SC, and a significant proportion of RGCs project only to the SC (Ellis et al., 2016). In addition, visual features are represented in the mouse SC, including motion direction and orientation (Wang et al., 2010; Ahmadlou and Heimel, 2015; Feinberg and Meister, 2015; Kay and Triplett, 2017; De Franceschi and Solomon, 2018), which are not present in the SC of primates but instead emerge in the visual cortex. Thus, the retinocollicular pathway in mice is responsible for much of the early visual processing that is associated with the retinogeniculatecortical pathway in primates.

Accordingly, although mouse primary visual cortex (V1) is important for visually guided behavior, it is not always essential, and the effects of V1 lesions depend on the particulars of the visual task. Using a water maze task, complete removal of mouse V1 reduces visual acuity by $\sim 50 \%$ for discriminating higher spatial frequency [ $>0.3$ cycles per degree (cpd)] gratings (Prusky and Douglas, 2004). However, the importance of V1 for visual detection and processing lower spatial frequencies is less clear. A recent study using low-spatial frequency gratings similar to ours $(\sim 0.1 \mathrm{cpd})$ found that optogenetic inhibition of mouse V1 during a visual foraging task caused deficits in orientation discrimination performance (Resulaj et al., 2018), but left the detection of gratings intact. In contrast, another study using more sensitive psychometric measurement showed that the inhibition of mouse V1 caused modest increases in detection thresholds for orientation or contrast changes (Glickfeld et al., 2013). Together, these results indicate that mouse V1 is important for discriminating visual features, but is less critical for visual detection especially at lower spatial frequencies.

In contrast, our results demonstrate that visual activity in the $\mathrm{SC}$ is crucial for detection performance in the mouse. The deficits caused by brief ( $100 \mathrm{~ms}$ ) SC inhibition in our task were similar to, if not larger than, those caused by much longer V1 inhibition (seconds) spanning the full trial duration (Glickfeld et al., 2013). In fact, the modest detection deficit caused by mouse V1 inhibition might be mediated through the SC, as the gain of visual responses in SC can be modulated by inputs from V1 (Zhao et al., 2014). Moreover, the effects of SC inhibition we observed exhibited remarkable temporal specificity. Detection performance was markedly impaired by inhibition applied during a temporal epoch that matched phasic visual activity in the SC, but not in the epochs immediately before or after. We do not conclude that SC activity in these other epochs is not important, since it is possible that deficits might emerge with stronger inhibition, but our results demonstrate the particular importance of visual activity in the SC for detection performance.

Another recent study also included optogenetic suppression of the mouse SC during a visual go/no-go task as part of a more expansive study examining the circuits linking prefrontal and visual cortex (Hu et al., 2019). As in our results, they found that SC inhibition caused by activation of GABAergic neurons in the SC caused significant changes in visual task performance. However, several details indicate that the two sets of findings are complementary rather than duplicative. Specifically, our psychometric analysis revealed changes in visual detection thresholds in addition to effects on lapse and guess rates, whereas $\mathrm{Hu}$ et al. (2019) mainly found changes in false alarm rates with their suprathreshold orientation difference (i.e., horizontal vs vertical gratings). Thus, our results provide evidence for changes in visual perceptual processing, in addition to possible changes in response bias or task engagement, and more generally illustrate the value of psychometric analysis.

Visual information processed in the mouse SC is also passed along to visual cortical areas. Outputs from mouse SC visual neurons can modulate visual response properties of neurons in V1 (Hu et al., 2019) and secondary visual cortex (Tohmi et al., 2014) through the lateral posterior nucleus of thalamus (Tohmi et al., 2014; Gale and Murphy, 2018; Zhou et al., 2018). Ascending projections from mouse SC appear to carry visual information distinct from V1 projections, particularly for visual motion. SC inhibition in mice has large effects on visual motion responses in postrhinal cortex (POR; Beltramo and Scanziani, 2019), a higher-order visual cortical area crucial for spatial navigation (LaChance et al., 2019), whereas inhibiting V1 has no effect on the motion response of POR neurons. In addition, SC lesions in mice also shift velocity tuning curves toward slower speeds in many secondary visual cortical areas (Tohmi et al., 2014). Thus, it is possible that the detection deficits caused by SC inhibition in 
our task, which used drifting visual gratings, could be due to disruptions in the processing of visual motion signals.

Alternatively, the perceptual deficits caused by SC inhibition could be due to a more generalized deficit in visual detection. Studies in primates have shown that SC inhibition causes deficits in detecting changes of visual features that are not represented by SC neurons (Herman et al., 2018). However, since neurons in the mouse SC and V1 are tuned to very similar visual features (Wang et al., 2010; Ito et al., 2017), to test whether the mouse SC serves as a general event detector would require additional experiments designed to test visual features that are not represented in the mouse SC.

\section{Superior colliculus and visual selection}

We found SC inhibition in mice caused larger deficits in detection when a competing visual stimulus was present in the other visual hemifield, consistent with the well established role for the SC in mediating visual competition and selective attention (Mysore and Knudsen, 2011; Krauzlis et al., 2013). For example, perceptual deficits caused by reversible inactivation of the primate SC are small when only a single visual stimulus is placed in the affected part of the visual field, but much larger when competing distractors are included elsewhere in the visual field (Lovejoy and Krauzlis, 2010, 2017). In contrast, although we found larger deficits with competing stimuli (our double-patch condition), we also found substantial deficits even with a single stimulus placed in the affected visual field.

One explanation for this difference between our results and previous findings in primate SC is the relative importance of the SC to the rest of the visual system. In contrast to mice, in monkeys the retinogeniculate-cortical route is the dominant visual pathway. The primate SC superficial layers receive retinal inputs only from $<10 \%$ of RGCs (Perry and Cowey, 1984), and most perceptual and saccade-related functions of primate SC involve the intermediate and deep layers, where visual activity signals behavioral priority within a retinotopic spatial map (Boehnke and Munoz, 2008; Basso and May, 2017). Although we mainly targeted GABAergic neurons in the SC intermediate layers, the strong GABAergic collateral projections from intermediate to superficial layers (Phongphanphanee et al., 2011) means that our optogenetic manipulation probably inhibited both superficial and deeper layers. The strong deficits in early visual processing caused by this inhibition probably limited our ability to measure effects on visual target selection. Given the complexity of intracollicular circuit organization, isolating effects on visual selection might require targeting either inhibitory inputs to the intermediate layers or subsets of intermediate layer GABAergic interneurons that lack axonal collaterals to the superficial layers. It might also be useful to adopt tasks that impose delays between the visual events and the motor response or that require the mice to integrate the sensory evidence over time.

Selecting relevant visual targets among competing distractors is an important aspect of visual selective attention. We previously demonstrated that mice can use spatial cues to improve their visual perceptual sensitivity and adjust their detection thresholds in tasks similar to those used in primates (Wang and Krauzlis, 2018). The involvement of the mouse SC in visual selection revealed in the current study suggests that the SC could be an important brain structure for controlling visual selective attention in mice.

\section{Conclusion}

Our results demonstrate that the SC in mice is necessary for the normal performance of visual perceptual decisions, in addition to its established role in mediating innate visual behaviors. The involvement of SC in our visual detection task was especially important during a 100-ms interval coincident with the phasic visual response of SC neurons. The larger deficits found in the presence of a competing visual stimulus are consistent with a role for the mouse SC in visual target selection. Thus, our study highlights the importance of the SC in mouse visual perception and illustrates the importance of the mouse SC as a model system to study neural mechanisms of voluntary visual choices.

\section{References}

Ahmadlou M, Heimel JA (2015) Preference for concentric orientations in the mouse superior colliculus. Nat Commun 6:6773.

Ahmadlou M, Zweifel LS, Heimel JA (2018) Functional modulation of primary visual cortex by the superior colliculus in the mouse. Nat Commun 9:3895.

Basso MA, May PJ (2017) Circuits for action and cognition: a view from the superior colliculus. Annu Rev Vis Sci 3:197-226.

Beltramo R, Scanziani M (2019) A collicular visual cortex: neocortical space for an ancient midbrain visual structure. Science 363:64-69.

Boehnke SE, Munoz DP (2008) On the importance of the transient visual response in the superior colliculus. Curr Opin Neurobiol 18:544-551.

Brainard DH (1997) The psychophysics toolbox. Spat Vis 10:433-436.

Carello CD, Krauzlis RJ (2004) Manipulating intent: evidence for a causal role of the superior colliculus in target selection. Neuron 43:575-583.

Danskin B, Denman D, Valley M, Ollerenshaw D, Williams D, Groblewski P, Reid C, Olsen S, Waters J (2015) Optogenetics in mice performing a visual discrimination task: measurement and suppression of retinal activation and the resulting behavioral artifact. PLoS One 10:e144760.

Dean P, Redgrave P (1984) The superior colliculus and visual neglect in rat and hamster. 1. Behavioural evidence. Brain Res 8:129-141.

Dean P, Redgrave P, Mitchell IJ (1988) Organisation of efferent projections from superior colliculus to brainstem in rat: evidence for functional output channels. In: Vision within extrageniculo-striate systems, Chap 3 (Hicks TP, Benedek G, eds), pp 27-36. Amsterdam: Elsevier.

De Franceschi G, Solomon SG (2018) Visual response properties of neurons in the superficial layers of the superior colliculus of awake mouse. J Physiol 596:6307-6332.

Dräger UC (1974) Autoradiography of tritiated proline and fucose transported transneuronally from the eye to the visual cortex in pigmented and albino mice. Brain Res 82:284-292.

Drager UC, Hubel DH (1976) Topography of visual and somatosensory projections to mouse superior colliculus. J Neurophysiol 39:91-101.

Eastman KM, Huk AC (2012) PLDAPS: a hardware architecture and software toolbox for neurophysiology requiring complex visual stimuli and online behavioral control. Front Neuroinform 6:1.

Ellis EM, Gauvain G, Sivyer B, Murphy GJ (2016) Shared and distinct retinal input to the mouse superior colliculus and dorsal lateral geniculate nucleus. J Neurophysiol 116:602-610.

Evans DA, Stempel AV, Vale R, Ruehle S, Lefler Y, Branco T (2018) A synaptic threshold mechanism for computing escape decisions. Nature 558:590-594.

Feinberg EH, Meister M (2015) Orientation columns in the mouse superior colliculus. Nature 519:229-232.

Franklin K, Paxinos G (2008) The mouse brain in stereotaxic coordinates. Amsterdam: Academic.

Gale SD, Murphy GJ (2018) Distinct cell types in the superficial superior colliculus project to the dorsal lateral geniculate and lateral posterior thalamic nuclei. J Neurophysiol 120:1286-1292.

Gandhi NJ, Katnani HA (2011) Motor functions of the superior colliculus. Annu Rev Neurosci 34:205-231.

Glickfeld LL, Histed MH, Maunsell JHR (2013) Mouse primary visual cortex is used to detect both orientation and contrast changes. J Neurosci 33:19416-19422.

Guo ZV, Inagaki HK, Daie K, Druckmann S, Gerfen CR, Svoboda K (2017) Maintenance of persistent activity in a frontal thalamocortical loop. Nature 545:181-186.

Herman JP, Katz LN, Krauzlis RJ (2018) Midbrain activity can explain perceptual decisions during an attention task. Nat Neurosci 21:1651-1660. 
Hofbauer A, Dräger UC (1985) Depth segregation of retinal ganglion cells projecting to mouse superior colliculus. J Comp Neurol 234:465-474.

Horwitz GD, Newsome WT (1999) Separate signals for target selection and movement specification in the superior colliculus. Science 284:11581161.

Hoy JL, Yavorska I, Wehr M, Niell CM (2016) Vision drives accurate approach behavior during prey capture in laboratory mice. Curr Biol 26:3046-3052.

Hu F, Kamigaki T, Zhang Z, Zhang S, Dan U, Dan Y (2019) Prefrontal corticotectal neurons enhance visual processing through the superior colliculus and pulvinar thalamus. Neuron 104:1141-1152.e4.

Huberman AD, Niell CM (2011) What can mice tell us about how vision works? Trends Neurosci 34:464-473.

Ito S, Feldheim DA, Litke AM (2017) Segregation of visual response properties in the mouse superior colliculus and their modulation during locomotion. J Neurosci 37:8428-8443.

Kay RB, Triplett JW (2017) Visual neurons in the superior colliculus innervated by Islet $2^{+}$or Islet $2^{-}$retinal ganglion cells display distinct tuning properties. Front Neural Circuits 11:73.

Krauzlis RJ, Miles FA (1996) Initiation of saccades during fixation or pursuit: evidence in humans for a single mechanism. J Neurophysiol 76:41754179.

Krauzlis RJ, Lovejoy LP, Zénon A (2013) Superior colliculus and visual spatial attention. Annu Rev Neurosci 36:165-182.

LaChance PA, Todd TP, Taube JS (2019) A sense of space in postrhinal cortex. Science 365:eaax4192.

Liu B-H, Huberman AD, Scanziani M (2016) Cortico-fugal output from visual cortex promotes plasticity of innate motor behaviour. Nature 538:383-387.

Lovejoy LP, Krauzlis RJ (2010) Inactivation of primate superior colliculus impairs covert selection of signals for perceptual judgments. Nat Neurosci 13:261-266.

Lovejoy LP, Krauzlis RJ (2017) Changes in perceptual sensitivity related to spatial cues depends on subcortical activity. Proc Natl Acad Sci USA 114:6122-6126.

Macmillan NA Creelman CD (2005) Detection theory: a user's guide, Ed 2. London: Erlbaum.

Mysore SP, Knudsen EI (2011) The role of a midbrain network in competitive stimulus selection. Curr Opin Neurobiol 21:653-660.

Pachitariu M, Steinmetz NA, Kadir S, Carandini M, Harris KD (2016) Kilosort: realtime spike-sorting for extracellular electrophysiology with hundreds of channels. bioRixv. doi:10.1101/061481.

Pelli DG (1997) The VideoToolbox software for visual psychophysics: transforming numbers into movies. Spat Vis 10:437-442.

Perry VH, Cowey A (1984) Retinal ganglion cells that project to the superior colliculus and pretectum in the macaque monkey. Neuroscience 12:1125-1137.

Phongphanphanee P, Mizuno F, Lee PH, Yanagawa Y, Isa T, Hall WC (2011) A circuit model for saccadic suppression in the superior colliculus. J Neurosci 31:1949-1954.

Pisupati S, Chartarifsky-Lynn L, Khanal A, Churchland AK (2019) Lapses in perceptual decisions reflect exploration. bioRxiv. doi:10.1101/613828.

Prusky GT, Douglas RM (2004) Characterization of mouse cortical spatial vision. Vision Res 44:3411-3418.
Resulaj A, Ruediger S, Olsen SR, Scanziani M (2018) First spikes in visual cortex enable perceptual discrimination. eLife 7:e34044.

Rossi MA, Li HE, Lu D, Kim IH, Bartholomew RA, Gaidis E, Barter JW, Kim N, Cai MT, Soderling SH, Yin HH (2016) A GABAergic nigrotectal pathway for coordination of drinking behavior. Nat Neurosci 19:742-748.

Sakatani T, Isa T (2004) PC-based high-speed video-oculography for measuring rapid eye movements in mice. Neurosci Res 49:123-131.

Schneider GE (1969) Two visual systems. Science 163:895-902.

Seabrook TA, Burbridge TJ, Crair MC, Huberman AD (2017) Architecture, function, and assembly of the mouse visual system. Annu Rev Neurosci 40:499-538.

Shang C, Liu Z, Chen Z, Shi Y, Wang Q, Liu S, Li D, Cao P (2015) A parvalbumin-positive excitatory visual pathway to trigger fear responses in mice. Science 348:1472-1477.

Shang C, Liu A, Li D, Xie Z, Chen Z, Huang M, Li Y, Wang Y, Shen WL, Cao $\mathrm{P}$ (2019) A subcortical excitatory circuit for sensory-triggered predatory hunting in mice. Nat Neurosci 22:909-920.

Sinex DG, Burdette LJ, Pearlman AL (1979) A psychophysical investigation of spatial vision in the normal and reeler mutant mouse. Vision Res 19:853-857.

Stahl JS (2004) Eye movements of the murine P/Q calcium channel mutant rocker, and the impact of aging. J Neurophysiol 91:2066-2078.

Tohmi M, Meguro R, Tsukano H, Hishida R, Shibuki K (2014) The extrageniculate visual pathway generates distinct response properties in the higher visual areas of mice. Curr Biol 24:587-597.

Wang L, Krauzlis RJ (2018) Visual selective attention in mice. Curr Biol 28:676-685.e4.

Wang L, Sarnaik R, Rangarajan K, Liu X, Cang J (2010) Visual receptive field properties of neurons in the superficial superior colliculus of the mouse. J Neurosci 30:16573-16584.

Wang L, Liu M, Segraves MA, Cang J (2015) Visual experience is required for the development of eye movement maps in the mouse superior colliculus. J Neurosci 35:12281-12286.

Wang L, Rangarajan KV, Gerfen CR, Krauzlis RJ (2018) Activation of striatal neurons causes a perceptual decision bias during visual change detection in mice. Neuron 97:1369-1381.e5.

Wei P, Liu N, Zhang Z, Liu X, Tang Y, He X, Wu B, Zhou Z, Liu Y, Li J, Zhang Y, Zhou X, Xu L, Chen L, Bi G, Hu X, Xu F, Wang L (2015) Processing of visually evoked innate fear by a non-canonical thalamic pathway. Nat Commun 6:6756.

Wichmann FA, Hill NJ (2001) The psychometric function: I. Fitting, sampling, and goodness of fit. Percept Psychophys 63:1293-1313.

Wurtz RH, Albano JE (1980) Visual-motor function of the primate superior colliculus. Annu Rev Neurosci 3:189-226.

Zénon A, Krauzlis RJ (2012) Attention deficits without cortical neuronal deficits. Nature 489:434-437.

Zhao X, Liu M, Cang J (2014) Visual cortex modulates the magnitude but not the selectivity of looming-evoked responses in the superior colliculus of awake mice. Neuron 84:202-213.

Zhou N, Masterson SP, Damron JK, Guido W, Bickford ME (2018) The mouse pulvinar nucleus links the lateral extrastriate cortex, striatum, and amygdala. J Neurosci 38:347-362. 